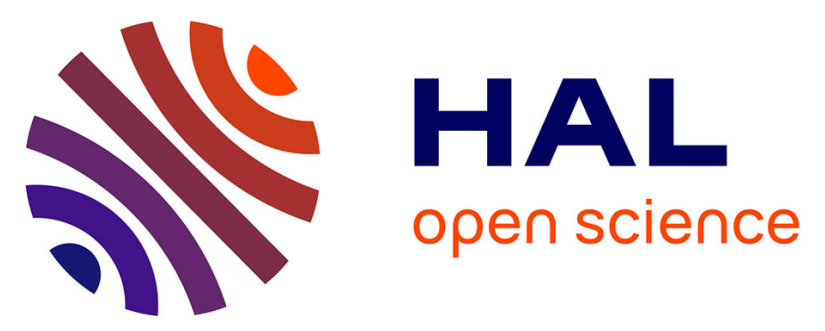

\title{
The HIV-1 ribonucleoprotein dynamically regulates its condensate behavior and drives acceleration of protease activity through membrane-less granular phase-separation
}

Sébastien Lyonnais, S Kashif Sadiq, Cristina Lorca-Oro, Laure Dufau, Sara Nieto-Marquez, Tuixent Escriba, Natalia Gabrielli, Xiao Tan, Mohamed Ouizougun-Oubari, Josephine Okoronkwo, et al.

\section{- To cite this version:}

Sébastien Lyonnais, S Kashif Sadiq, Cristina Lorca-Oro, Laure Dufau, Sara Nieto-Marquez, et al.. The HIV-1 ribonucleoprotein dynamically regulates its condensate behavior and drives acceleration of protease activity through membrane-less granular phase-separation. 2019. hal-02349017

\author{
HAL Id: hal-02349017 \\ https://hal.science/hal-02349017
}

Preprint submitted on 5 Nov 2019

HAL is a multi-disciplinary open access archive for the deposit and dissemination of scientific research documents, whether they are published or not. The documents may come from teaching and research institutions in France or abroad, or from public or private research centers.
L'archive ouverte pluridisciplinaire HAL, est destinée au dépôt et à la diffusion de documents scientifiques de niveau recherche, publiés ou non, émanant des établissements d'enseignement et de recherche français ou étrangers, des laboratoires publics ou privés. 


\section{The HIV-1 ribonucleoprotein dynamically regulates its condensate behavior and drives acceleration of protease activity through membrane-less granular phase- separation}

Sébastien Lyonnais ${ }^{1,2} \#^{*}$, S. Kashif Sadiq ${ }^{3,4} \#^{*}$, Cristina Lorca-Oró ${ }^{1}$, Laure Dufau ${ }^{5}$, Sara NietoMarquez $^{1}$, Tuixent Escriba ${ }^{1}$, Natalia Gabrielli ${ }^{1}$, Xiao Tan $^{1,5}$, Mohamed Ouizougun-Oubari ${ }^{1}$, Josephine Okoronkwo ${ }^{1}$, Michèle Reboud-Ravaux ${ }^{5}$, José Maria Gatell ${ }^{1,6}$, Roland Marquet ${ }^{7}$, JeanChristophe Paillart ${ }^{7}$, Andreas Meyerhans ${ }^{3,8}$, Carine Tisné ${ }^{9}$, Robert J. Gorelick ${ }^{10} \&$ Gilles Mirambeau $^{1,11, \# *}$

${ }^{1}$ Infectious disease \& AIDS Research Unit, IDIBAPS, Villaroel 170, Barcelona, Spain.

${ }^{2}$ CEMIPAI, UMS 3725 Université de Montpellier - CNRS, 1919 Route de Mende, 34000 Montpellier.

${ }^{3}$ Infection Biology Laboratory, DCEXS, Universitat Pompeu Fabra, Barcelona, Spain.

${ }^{4}$ Molecular and Cellular Modeling Group, Heidelberg Institute for Theoretical Studies (HITS), Schloss-Wolfsbrunnenweg 35, 69118 Heidelberg, Germany

${ }^{5}$ UMR/CNRS 8256, IBPS, Sorbonne Universités, UPMC Univ Paris 06, Paris, France.

${ }^{6}$ Infectious Disease and HIV Service, Hospital Clínic, University of Barcelona, Barcelona, Spain.

${ }^{7}$ Architecture et Réactivité de l'ARN, CNRS, Université de Strasbourg, Institut de Biologie Moléculaire et Cellulaire, Strasbourg, France.

${ }^{8}$ Institució Catalana de Recerca i Estudis Avançats (ICREA), Barcelona, Spain.

${ }^{9}$ Laboratoire de Cristallographie et RMN Biologiques, CNRS, Paris Sorbonne Cité, 4 Avenue de l'Observatoire, Paris, 75006, France.

${ }^{10}$ AIDS and Cancer Virus Program, Leidos Biomedical Research, Inc., Frederick National Laboratory for Cancer Research, Frederick, Maryland, USA.

11 UFR 927 des Sciences de la Vie, Faculté des Sciences et d'Ingénierie, Sorbonne Universités, Paris, France.

\# These authors contributed equally.

*Correspondence: sebastien.lyonnais@cemipai.cnrs.fr, kashif.sadiq@h-its.org, gilles.mirambeau@upmc.fr 


\begin{abstract}
A growing number of studies indicate that mRNAs and long ncRNAs can affect protein populations by assembling dynamic ribonucleoprotein (RNP) granules. These phaseseparated molecular 'sponges', stabilized by quinary (transient and weak) interactions, control proteins involved in numerous biological functions. Retroviruses such as HIV-1 form by self-assembly when their genomic RNA (gRNA) traps Gag and GagPol polyprotein precursors. Infectivity requires extracellular budding of the particle followed by maturation, an ordered processing of $\sim 2400 \mathrm{Gag}$ and $\sim 120 \mathrm{GagPol}$ by viral protease (PR). This leads to a condensed gRNA-NCp7 nucleocapsid and a CAp24-self-assembled capsid surrounding the RNP. The choreography by which all of these components dynamically interact during virus maturation is one of the missing milestones to fully depict the HIV life cycle. Here, we describe how HIV-1 has evolved a dynamic RNP granule with successive weak-strong-moderate quinary NC-gRNA networks during the sequential processing of the GagNC domain. We also reveal two palindromic RNA-binding triads on NC, KxxFxxQ and QxxFxxK, that provide quinary NC-gRNA interactions. Consequently, the nucleocapsid complex appears properly aggregated for capsid reassembly and reverse transcription, mandatory processes for viral infectivity. We show that PR is sequestered within this RNP and drives its maturation/condensation within minutes, this process being most effective at the end of budding. We anticipate such findings will stimulate further investigations of quinary interactions and emergent mechanisms in crowded environments throughout the wide and growing array of RNP granules.
\end{abstract}

\title{
Introduction
}

In response to cellular demands and physiological stresses', eukaryotes have evolved mechanisms that sequester mRNAs and associated RNA-binding proteins into membrane-less organelles called RNA granules. Such self-assemblies form gel phases in the cell cytoplasm, which exhibit adaptable dynamic architectures depending on their composition and biological functionality ${ }^{2}$. The concept of biological glue has been suggested previously, such as in a crowded environment, the sum of many weak, termed quinary interactions, can promote the assembly of highly stable, but dynamic and transient multi-macromolecular complexes without any requirement for membrane compartmentalization ${ }^{3,4}$.

Even though an HIV-1 particle is derived from the self-assembled $\mathrm{P} 55^{\mathrm{Gag}}$ shell and is ultimately enveloped by a lipid membrane, the concept of quinary interactions is clearly applicable in describing its dynamic assembly at the mesoscopic scale-since it forms a confined 
RNP gel phase in a highly crowded space, within a limited time frame and in a cooperative manner. P55 ${ }^{\mathrm{Gag}}$ is composed from $\mathrm{N}$ - to C-termini of matrix (MAp17), capsid (CAp24), spacer peptide SP1, nucleocapsid (NC), spacer peptide SP2 and p6 protein. Key players here consist of NC protein intermediates with their variable nucleic acid (NA) binding properties that are dependent upon their processing state ${ }^{5-8}$. Tethered within the virion by approximately 2400 GagNC domains, the two single strands of $9.2 \mathrm{~kb}$-long gRNA specifically scaffold P55 ${ }^{\text {Gag }}$ selfassembly. Subsequently, the HIV-1 RNP complex engages a granular condensation during the sequential proteolysis of the P55 ${ }^{\mathrm{Gag}}$ RNA-binding domain into the mature nucleocapsid protein (NCp7) by the viral protease (PR $)^{7,9,10}$. PR is derived by autoprocessing of a smaller number of GagPol within the P55 ${ }^{\mathrm{Gag}}$ assembly that additionally contain reverse transcriptase (RT) and integrase (IN). Approximately $60 \mathrm{PR}$ homodimers are potentially available to catalyze maturation, which principally requires 12000 cleavage events. Cleavage of GagNC by PR generates first NCp15 (NCp7-SP2-p6) bound to, and forming with the gRNA an RNP intermediate that physically detaches from the remaining outer MA-CA-SP1 shell. The second cleavage between SP2 and p6 releases NCp9 (NCp7-SP2) (Fig. 1a, Fig. 6b). Single-stranded nucleic acids (ssNA) stimulate both cleavage events in vitro ${ }^{6,11,12}$. The third cleavage produces the mature 55 amino acids (aa)-long NCp7 and SP2. Within the virus, NCp15 seems to condense gRNA less well than $\mathrm{NCp} 9$ and $\mathrm{NCp} 7^{13}$. Yet $\mathrm{NCp} 9$ does not appear as functional as $\mathrm{NCp} 7^{14}$. NCp15 and NCp9 are short-lived species not detected during typical virus production ${ }^{8}$. Why such intermediates are maintained along the HIV-1 maturation process remains unclear.

HIV-1 PR is an aspartyl-protease, enzymatically active only as a homodimer. Recombinant $\mathrm{PR}$ is stabilized in vitro by high ionic strength $(>1 \mathrm{M} \mathrm{NaCl})$ and catalysis is strongly activated under acidic conditions (pH 5.0 or even lower). Lower salt $(0.1 \mathrm{M} \mathrm{NaCl})$ and increasing the $\mathrm{pH}$ to 6.0 limits the acidic catalysis and shifts the equilibrium towards the monomer ${ }^{15}$. At quasi-neutral $\mathrm{pH}$, in low salts and an excess of $\mathrm{PR}$, the in vitro cleavage of Gag follows the sequential mechanism described above leading to $\mathrm{NCp} 7$ and the condensed $\mathrm{RNP}^{16}$. RNA or ssDNA promote NCp15 cleavage in vitro ${ }^{6,17}$, while recent reports have shown that direct RNAPR contacts enhance the enzyme activity ${ }^{12}$. Consequently, PR appears to engage in an intricate partnership with $\mathrm{NC}$ and gRNA during viral maturation that remains incompletely understood. HIV-1 NCp7 contains a small globular domain formed with two zinc fingers (ZFs) that generate a hydrophobic pocket with two aromatic residues (Phe16 and Trp37). This platform stacks with unpaired nucleotides, preferentially guanosines exposed in RNA or ssDNA secondary structures, while basic residues stabilize the complex through electrostatic interactions with the NA backbone. With particular stem-loops in gRNA or DNA, this results in the formation of specific complexes ${ }^{18,19}$. NCp7 is also a highly mobile and flexible polycationic condensing agent; like polyamines, transient protein:NA electrostatic contacts neutralize phosphate backbone repulsions lowering the overall energy of the RNP complex ${ }^{7,20,21}$. In vitro, these properties induce a massive 
co-aggregation of recombinant $\mathrm{NCp} 7$ and $\mathrm{NCp} 9$ with $\mathrm{NA}$ templates ${ }^{5,8,22}$. This "molecular gluing" capability guides the matchmaking/NA chaperone activity by facilitating intra- and intermolecular RNA-RNA interactions required for functional gRNA folding ${ }^{23}$. Such crowding effects rely on basic residues particularly concentrated in the two small flexible domains, the (114) N-terminal domain and the (29-35) linker between the $\mathrm{ZFs}^{20}$. NCp15 shows slightly different NA binding and chaperone properties but is essentially characterized by a reduced ability to aggregate $\mathrm{NA}^{5,8,24}$, properties recently correlated with a direct fold-back contact between the $\mathrm{p} 6$ and ZF domains ${ }^{8}$. NCp9 shows an enhanced NA affinity due to a slower dissociation rate, as well as dramatically enhanced NA aggregating activities ${ }^{5,8,21}$. Alanine substitution of acidic residues in $\mathrm{p} 6$ converts $\mathrm{NCp} 15$ to a NA-aggregating protein similar to NCp9, while addition of a p6 peptide lowers the RNA chaperone activity of $\mathrm{NCp} 7$ in vitro ${ }^{8}$. This suggests that $\mathrm{SP} 2$ contains an additional NA-interaction domain, which may be masked or modulated with another NCp7 domain by intra- or intermolecular protein contacts between $\mathrm{p} 6$ and the $\mathrm{NC}$ domain.

HIV-1 maturation is mandatory for viral dissemination following sequential processes of protein and RNA self-assembly, coordinated in space and time by the enzymatic activity of viral $\mathrm{PR}^{9,10,25}$. The slow in vitro kinetics of Gag proteolysis supports a general scheme for PR to be auto-processed during the completion of budding thus driving viral maturation within free, released particles in a computed time-scale close to $30 \mathrm{~min}^{26}$. This model is, however, inconsistent with many observations from electron microscopy that shows i) a huge majority of free but freshly released particles in a mature form containing condensed $\mathrm{RNP}^{27}$, ii) both capsid and budding defects in presence of PR inhibitors ${ }^{28}$, and iii) budding and maturation defects for critical NC mutants, whereas Western blots from cell extracts detects PR-processed Gag products ${ }^{27}$. Such findings suggest a much closer overlap between budding and maturation than generally supposed. Importantly, suppressing both PR cleavage sites in NCp15 abolishes viral infectivity ${ }^{13,29}$ and results in an abnormal virion core morphology ${ }^{13}$. In contrast, suppression of the NCp7-SP2 cleavage site shows little effect on virus morphology and infectivity in single-cycle assays, but reverts to WT (e.g. containing NCp7) after several rounds of infection ${ }^{29}$. A "roadblock" mechanism affecting RT activity on a NA template has been shown to be imparted by NCp9 as well as by $\mathrm{NCp} 15$, which could limit large-scale viral replication, highlighting $\mathrm{NCp} 7$ as the optimized cofactor for accurate RNP folding and viral fitness ${ }^{14}$.

The present study highlights how HIV-1 gRNA becomes condensed by NC proteins through the action of the RNP-sequestered PR enzyme. Reconstituted systems that model non sequence-specific binding on a large scale allowed us i) to detail the quinary effects and their variations engaged in this dynamic process as well as ii) to focus on PR action in such a gluing context. 


\section{METHODS}

\section{Proteins, Nucleic Acids and Reagents.}

Proteins. The HIV-1 NC proteins and proviral plasmids were based on the pNL4-3 sequence (GenBank accession number AF324493). Recombinant wild-type and mutants of NCp7, NCp9 and NCp15, respectively 55, 71 and 123 amino acids in length, were expressed and purified as described $^{8,30-32}$. The CA-SP1-NC-SP2-p6 protein expression construct was generated by PCR amplifying pNL4-3 using Gag $\triangle$ MA sense primer 5'-GAT CTG GGT ACC GAG AAC CTC TAC TTC CAG ATG ATA GTG CAG AAC, NL43 OCH antisense primer 5'-GCT TGA ATT CTT ATT GTG ACG AGG GGT CGC TGC and cloning the resulting product into the homologous KpnI and EcoRI sites of pET32a (Novagen, Madison, WI). Expression construct for NCp15(1) (expressing NCp15 that can be cleaved only to NCp9) and NCp15(2) (expressing uncleavable NCp15) were generated starting with the NC-SP2- and NCp15-containing proviral plasmids of Coren et al. ${ }^{29}$, respectively. The two constructs were generated by PCR amplifying the appropriate plasmids with NL4-3 NC sense primer 5'-CGT GGG ATC CTT AGA GAA CCT CTA CTT CCA GAT ACA GAA AGG CAA TTT TAG, NL4-3 NCp15 antisense primer 5'-GTA CGT GTC GAC TCT CTA ATT ATT GTG ACG AGG GGT CGC T and cloning into the homologous BamHI and SalI sites of pET32a. Site-directed mutagenesis of the wild-type NL4$3 \mathrm{NCp} 7$ construct to generate the K3A/F6A/Q9A mutant was performed using the Agilent QuickChange Site-Directed Mutagenesis kit, with verification by NA sequence analysis, for the generation of the recombinant expression plasmid, as described ${ }^{33}$. The K3A mutation results from changes to nucleotides 1927 through 1929 from AAA to GCC, F6A results from nucleotides 1936 and 1937 being changed from TT to GC, and Q9A results from nucleotides 1945 and 1946 being changed from CA to GC. Proteins were expressed and purified as described ${ }^{8,30-32}$. Proteins were stored lyophilized and then suspended at a concentration of $1 \mathrm{mg} / \mathrm{mL}$ in a buffer containing $20 \mathrm{mM}$ HEPES pH7.5, $50 \mathrm{mM}$ sodium acetate, $3 \mathrm{mM}$ DTT, 20\% (v/v) ethylene glycol, $200 \mu \mathrm{M}$ $\mathrm{ZnCl}_{2}$ and stored at $-20^{\circ} \mathrm{C}$. The concentrations were determined by measuring the $\mathrm{UV}$ absorbance at $280 \mathrm{~nm}$ using the following extinction coefficients: $\mathrm{NCp} 7$ and $\mathrm{NCp} 7$ mutants: $5690 \mathrm{M}^{-1}$. $\mathrm{cm}^{-1}$;

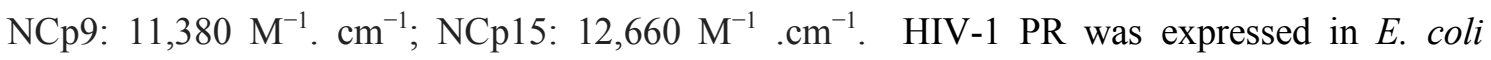
Rosetta(DE3)pLysS strain (Novagen) as inclusion bodies using the expression vector pET-9 and purified as described ${ }^{34,35}$. The PR domain used here bears the Q7K/L33I/L63I and C67A/C95A protective mutations to respectively minimize autoproteolysis ${ }^{15}$ and prevent cysteine-thiol oxidation $^{36}$. PR was suspended, adjusted to $10-20 \mu \mathrm{M}$ concentration and stored at $-80^{\circ} \mathrm{C}$ in 50 $\mathrm{mM}$ sodium acetate $\mathrm{pH} 5.5,100 \mathrm{mM} \mathrm{NaCl}, 1 \mathrm{mM}$ DTT, $0.1 \mathrm{mM}$ EDTA, 10\% (v/v) glycerol.

Nucleic Acids. The circular 7,249 nt M13 ssDNA (m13mp18) was purchased from Bayou Biolabs, the 3,569 nt MS2 RNA from Roche GmBh. Linear m13mp18 molecules were generated 
by annealing a complementary oligonucleotide to form a restriction site for BsrB I (NEB) as described $^{37}$. The oligonucleotides poly $\mathrm{d}(\mathrm{A})_{13}$ and TAR-RNA (27 nt, 5'CCAGAUCUGAGCCUGGGAGCUCUCUGG-3'), were purchased from Sigma-Aldrich, the short RNA fragments corresponding to individual stem-loop motifs of the Psi region: SL1 (17 nt), SL2 (23 nt), SL3 (14 nt) and SL4 (24 nt) were purchased (Microsynth) and purified by HPLC (ÄKTA design-Unicorn) on a PA-100 anion exchange column (Dionex). Plasmids used for in vitro transcription of HIV-1 RNAs used in this study have been described previously ${ }^{38,39}$. Briefly, the pJCB vector was linearized with AflII, XbaI, BssHII, RsaI, or PvuII, and used as templates for the synthesis of RNAs 1-61, 1-152, 1-278, 1-311 and 1-615, respectively, by in vitro run off transcription using bacteriophage T7 RNA polymerase, followed by purification using size exclusion chromatography as described previously ${ }^{40}$. Likewise, plasmid pmCG67 was linearized with AvaII or SalI to produce RNAs 1-1333 and 1-4001, respectively. RNA 1-102 was obtained from a PCR product corresponding to the HIV-1 MAL sequence.

\section{NP complex assembly and electrophoretic mobility shift assay}

Unless stated otherwise, electrophoretic mobility shift assays (EMSA) contained $1 \mathrm{ng} / \mu \mathrm{L}$ NA templates $(0.4 \mathrm{nM})$ in $20 \mu \mathrm{L}$ and were performed at $37^{\circ} \mathrm{C}$ in a binding buffer containing $20 \mathrm{mM}$ Tris-HCl pH 7.4, $1 \mathrm{mM} \mathrm{MgCl} 2,100 \mathrm{mM} \mathrm{NaCl}, 1 \mathrm{mM}$ DTT. NCp were diluted on ice in the reaction buffer. For reactions using NCp15, the buffers were supplemented with $0.1 \%(\mathrm{w} / \mathrm{v})$ Tween 20. Reactions were initiated by addition of $\mathrm{NC}$ as appropriate and terminated at $30 \mathrm{~min}$. (unless stated otherwise) by chilling the tubes on ice and addition of $10 \%(\mathrm{v} / \mathrm{v})$ a loading buffer (30\% glycerol, $0.01 \%$ xylene cyanol, $10 \mathrm{mM}$ Tris-HCl pH 7.4). Samples were then fractionated on $25 \mathrm{~cm}$-long, $1 \%$ (w/v) agarose gels (SeaKem LE Agarose, Lonza) in 0.5x TBE. Gels were run in a Sub-Cell GT cuvette (BioRad) for 17-18 hr at room temperature at $3 \mathrm{~V} / \mathrm{cm}$, stained with SybrGold (Molecular Probes) and scanned for fluorescence using a Typhoon 8600 (GE Healthcare). All experiments were performed at least in triplicate.

\section{Dynamic Light Scattering}

Dynamic light scattering (DLS) measurements were carried out in $20 \mathrm{mM}$ Tris-Acetate $\mathrm{pH}$ 7.4, $50 \mathrm{mM}$ sodium acetate and $1 \mathrm{mM}$ DTT, using $2.5 \mathrm{ng} / \mu \mathrm{L} \mathrm{M} 13$ ssDNA. Experiments were performed with a Zetasizer Nano-ZS (Malvern Instruments Ltd) and high precision cells (QS 3.0 $\mathrm{mm}$, Hellma Analytics). Measurements were performed at $37^{\circ} \mathrm{C}, 20 \mathrm{~min}$ after addition of the indicated amount of NCp and analyzed by the Dispersion Technology Software provided.

\section{AFM imaging}

NC:NA complexes were assembled under conditions used for EMSA with $1 \mathrm{ng} / \mu 1$ of M13 ssDNA 
and in a binding solution containing $10 \mathrm{mM}$ TrisAcetate $\mathrm{pH} 7.0,50 \mathrm{mM}$ sodium acetate, 2.5 to 5 $\mathrm{mM}$ magnesium diacetate and $0.5 \mathrm{mM}$ TCEP. A freshly cleaved muscovite mica surface was pretreated for 2 min with a fresh dilution of spermidine $(50 \mu \mathrm{M})$, extensively rinsed with water and dried under a nitrogen flow ${ }^{41}$. A $5 \mu \mathrm{L}$-drop of the NP complexes was deposited on the surface and incubated for 3-5 min and dried with nitrogen gas. AFM images were carried out in air with a multimode scanning probe microscope (Bruker) operating with a Nanoscope IIIa or V controller (Bruker) and silicon AC160TS cantilevers (Olympus) using the tapping mode at their resonant frequency. The scan frequency was typically $1.0 \mathrm{~Hz}$ per line and the modulation amplitude was a few nanometers. A second order polynomial function was used to remove the background with the AFM software.

\section{Proteolysis assays}

\section{NC cleavage and SDS-PAGE analysis.}

A proteolysis assay of NCp15 bound to ssDNA using recombinant PR was showed previously ${ }^{6}$. The assay was optimized in this study to ensure a detailed analysis of the reaction using SDSPAGE electrophoresis (Supplementary Fig. 3a-b). Peptides were quantified by fluorescent staining, which allowed accurate measurements in the 25-500 ng range, in agreement with our NP complex analysis. The standard proteolysis assay contained NCp proteins $(6 \mu \mathrm{M})$ incubated with NA for $5 \mathrm{~min}$ at $37^{\circ} \mathrm{C}$ in $10 \mu \mathrm{l}$ of a PR buffer (MES $50 \mathrm{mM} /$ Tris variable to adjust $\mathrm{pH}, \mathrm{NaCl}$ $100 \mathrm{mM}$, DTT $2 \mathrm{mM}$, BSA $50 \mu \mathrm{g} / \mathrm{ml}$ ). Next, PR was added, unless otherwise indicated, at a concentration of $600 \mathrm{nM}$. Reactions were stopped by addition of a SDS-PAGE loading buffer and heat denaturation $\left(5 \mathrm{~min}\right.$ at $\left.95^{\circ} \mathrm{C}\right)$, followed by $1 \mathrm{~h}$ incubation at $37^{\circ} \mathrm{C}$ in presence of $300 \mathrm{mM}$ Iodoacetamide, which prevented protein oxidation (Supplementary Fig. 3a). Samples were separated on 20\% acrylamide gels using Tris-Tricine SDS-PAGE in a Hoeffer MiniVE system. After migration at $160 \mathrm{~V}$ for $2.5 \mathrm{hr}$, the gels were fixed by $40 \%$ ethanol/10\% acetic acid for $1 \mathrm{hr}$ and stained overnight in $200 \mathrm{~mL}$ of Krypton Fluorescent gel stain (Life Technologies) diluted $1 / 10$ in water. Gels were then rinsed with $5 \%$ acetic acid and incubated in milliQ water for $30 \mathrm{~min}$ before scanning with a Typhoon 8600 imager. Fluorescence counts were quantified using the ImageQuant software (GE Healthcare). Apparent Vmax was measured by dividing the product concentration by the time of incubation with [product]/ [ $\mathrm{S}_{0}$ ] product ratio less than $30 \%$. The PR cleavage assay of Fig. 1e-f was performed by incubating NCp15 (750 nM) and M13 ssDNA $(1 \mathrm{nM})$ in MES $50 \mathrm{mM} /$ Tris pH6.25, $100 \mathrm{mM} \mathrm{NaCl}, 4 \mathrm{mM} \mathrm{MgCl}_{2}, 2 \mathrm{mM}$ DTT for $15 \mathrm{~min}$ at $37^{\circ} \mathrm{C}$ in $50 \mu \mathrm{L}$. PR ( $35 \mathrm{nM})$ was added and the cleavage was carried out at the indicated times. Each reaction was stopped by chilling the tubes on ice while a $5 \mu 1$-drop was used to prepare mica for AFM, $15 \mu$ l were loaded on the gel for EMSA and the remaining $30 \mu 1$ were used for SDS-PAGE 
after treating the samples as previously indicated. All experiments were performed at least in triplicate.

FRET assay. The proteolytic activities of PR were determined using the principles of Förster resonance energy transfer (FRET) by cleavage of a fluorogenic peptide substrate DABCYL- $\gamma$ abu-Ser-Gln-Asn-Tyr-Pro-Ile-Val-Gln-EDANS (Bachem, Germany) with DABCYL, 4-(4'-dimethylaminophenylazo)benzoyl; $\gamma$-abu, $\gamma$-aminobutyric acid; EDANS, 5-[(2-aminoethyl)amino] naphthalene-1-sulfonic acid. Incubation of PR with the probe resulted in specific cleavage at the Tyr-Pro bond and a time-dependent increase in fluorescence intensity that is linearly related to the extent of substrate hydrolysis. Kinetic experiments were carried out at $30^{\circ} \mathrm{C}$ in $150 \mu \mathrm{L}$ of the PR buffer (50 mM MES-Tris combination, $0.1-1 \mathrm{M} \mathrm{NaCl}$, pH adjusted between 5 and 7), 5.2 $\mu \mathrm{M}$ of the probe and 10-50 nM of PR. The probe was first dissolved in DMSO. The final DMSO concentration was kept at $3 \%(\mathrm{v} / \mathrm{v})$. Fluorescence intensities were measured in a BMG Fluostar microplate reader. Delay time for the reaction start was calculated as the reaction slope intercept with the $\mathrm{x}$ axis. All experiments were performed at least in triplicate.

\section{Electron microscopy of HIV-1 particles}

Maturation mutants. Mutant virions accumulating $\mathrm{NCp} 15$ or $\mathrm{NCp} 9$ were produced by transfection of mutated pNL4-3 proviral plasmids as described ${ }^{29}$. Plasmids were transfected into HEK 293T cells using Mirus TransIT 293 (Mirus Bio LLC, Madison, WI) according to the manufacturer's instructions. $48 \mathrm{~h}$ culture supernatants were clarified and virions ultracentrifuged and examined by electron microscopy as described previously ${ }^{42,43}$. At least 180 particles were analysed on the criteria that they were enveloped and a contrast was visible inside. Then the subpopulation of the diffuse cores instead of thin, dark spots was scored with, as discriminating criteria, a diameter equal or larger than $70 \%$ of the internal diameter of the particle.

Viral particles produced from latently infected cells. Briefly, the latently infected $\mathrm{ACH} 2$ cells * were grown under standard conditions, were plated onto $10 \mathrm{~cm}$ cell culture dishes at densities of $4 \mathrm{x} 10^{\circ}$ cells and incubated with or without PR inhibitor (10 $\mu \mathrm{M}$ Lopinavir, Sigma). HIV production was activated by adding Vorinostat (10 $\mu \mathrm{M}$; Sigma). After 2 days, ACH2 cells were fixed with $2.5 \%$ glutaraldehyde, washed, dehydrated, embedded in epoxy resin according to standard procedures ${ }^{4}$. Electron microscopy images were obtained with a Tecnai Spirit microscope coupled with a 1376 x 1024 pixel CCD camera (FEI, Eindhoven, The Netherlands). We analysed 500 particles attached to the membranes after normal production and 120 after production in presence of Lopinavir (respectively 46.1 and 53\% of the total number of detectable particles). Within each attached population, mainly $91 \%$ particles were identifiable, $89.6 \%$ containing a dark spot compared to only $1.4 \%$ immature for the normal population, while dark spots were not visible after viral production in presence of Lopinavir. 


\section{Molecular Dynamics Simulations and Analysis}

Molecular dynamics simulations followed a previously well-established protocol ${ }^{46}$. An initial structure was prepared for the NC-SP2 (RQAN-FLGK) octapeptide ligand in apo-form. Atomic coordinates for the octapeptide were extracted from the 1TSU crystal structure ${ }^{46,47}$. The standard AMBER forcefield (ff03) ${ }^{48}$ was used to describe all parameters. The system was solvated using atomistic TIP3P water and then electrically neutralized with an ionic concentration of $0.15 \mathrm{M}$, resulting in a fully atomistic, explicit solvent system of approximately 14,000 atoms. Conjugategradient minimization was performed. The SHAKE algorithm was employed on all atoms covalently bonded to a hydrogen atom. The long range Coulomb interaction was handled using a GPU implementation of the particle mesh Ewald summation method (PME). A non-bonded cutoff distance of $9 \AA$ was used with a switching distance of $7.5 \AA$. During equilibration the position of all heavy peptide atoms was restrained by a $0.5 \mathrm{kcal} / \mathrm{mol} / \AA^{2}$ spring constant for all heavy protein atoms and the system evolved for $10 \mathrm{~ns}$ with a timestep of 4 fs. The temperature was maintained at $300 \mathrm{~K}$ using a Langevin thermostat with a low damping constant of $0.1 / \mathrm{ps}$ and the pressure maintained at $1 \mathrm{~atm}$ for both systems. The system was then equilibrated for $10 \mathrm{~ns}$ of unrestrained simulation in the canonical ensemble (NVT) with an integration timestep of $4 \mathrm{fs}$. The final coordinates were used as input for production simulations. All subsequent simulations were carried out in the NVT ensemble. All production simulations were carried out using $\mathrm{ACEMD}^{49}$. An ensemble of $10 \times 1 \mu$ s production simulations was performed. Coordinate snapshots from production simulations were generated every $10 \mathrm{ps}$, resulting in a ensemble of $10^{6}$ conformers for analysis.

The octapeptide was relabelled as R52-Q53-A54-N55-F56-L57-G58-K59. The conformer ensemble was analyzed in a reaction coordinate space consisting of two order parameters: the K59-Q53 $\mathrm{C}_{\alpha}$ distance $\left(\mathrm{d}_{\mathrm{KQ}}\right)$ and $\mathrm{K} 59-\mathrm{F} 56-\mathrm{Q} 53 \mathrm{C}_{\alpha}$ angle $\left(\theta_{\mathrm{KFQ}}\right)$. The potentials of mean force (PMF) was calculated by binning the ensemble data into microstates corresponding to the given reaction coordinate space and then calculating the mole fraction $(\rho)$ of each microstate using PMF $=-\mathrm{k}_{\mathrm{B}} \mathrm{T} \ln (\rho)$, where $\mathrm{k}_{\mathrm{B}}$ is the Boltzmann constant and $\mathrm{T}$ the temperature. Corresponding order parameters were calculated for each of the NMR conformers in PDB 1F6U of the NC N-terminus where $\mathrm{d}_{\mathrm{KQ}}$ was the K3-Q10 $\mathrm{C}_{\alpha}$ distance and the $\theta_{\mathrm{KFQ}} \mathrm{K} 3-\mathrm{F} 6-\mathrm{Q} 9 \mathrm{C}_{\alpha}$ angle. Conformers were then aligned to the NC N-terminus from PDB 1F6U by the $\mathrm{C}_{\alpha}$ atoms of K59-R52 mapped to K3-R10 of $1 \mathrm{~F} 6 \mathrm{U}$. The $\mathrm{C}_{\alpha}$ RMSD was then calculated as a third order parameter $\left(\mathrm{d}_{\mathrm{n}}\right)$, its probability density was determined by binning $\left(\rho\left(\mathrm{d}_{\mathrm{n}}\right)\right)$ and conformers within the thresholds of $1 \AA, 1.5 \AA$ and $2 \AA$ extracted and mapped back to the $\mathrm{d}_{\mathrm{KQ}}-\theta_{\mathrm{KFQ}}$ reaction coordinate space. 


\section{Results}

We first focused on the quinary interactions and the architectural behavior of NC:NA complexes by a combination of electrophoretic mobility shift assay in agarose gels (EMSA), atomic force microscopy (AFM) and dynamic light scattering (DLS). Examining RNPs with large ssNA templates under increasingly dilute conditions interestingly switched $\mathrm{NCp} 7$ binding from NA aggregation (quinary interactions) to intramolecularly-folded NP condensates (Fig. 1b). NCp7 binding titrations on a circular M13 ssDNA showed a progressive process of ssDNA migration acceleration in a gel (Fig. 1c), seen by AFM as tightly compact NP structures formed of folded DNA strands coated and bridged with protein (Fig. 1d). Maximum ssDNA compaction was reached for saturating amounts of one $\mathrm{NCp} 7$ over $8-10 \mathrm{nt}^{22}$. Additional protein resulted in the fusion of these NP condensates into very high molecular weight structures that exhibited smearing during electrophoresis. AFM showed a progressive accumulation of protein clusters covering the lattices while the branched and secondary structures of the ssDNA appeared melted or absent, and rather bridged into nucleofilament-like structures (Supplementary Fig.2). Omission of magnesium in the buffer (Fig. 2g-i) or an excess of $\mathrm{NCp} 7$ resulted in fusion of the individual condensates into huge macrostructures with a spheroid shape comparable with previously described NC:NA aggregates ${ }^{5,6,21}$. NCp7 mobility was deemed necessary since this fusion was not observed and condensation was delayed at $4{ }^{\circ} \mathrm{C}^{24,31}$ (Supplementary Fig 1a). The kinetics of the reaction indicated fast intramolecular condensation and a slow process of NP condensate fusion (Supplementary Fig. 1b). Low monovalent salt concentration increased NCp7/ssDNA aggregation and a strong electrostatic competition was observed with $\mathrm{Na}^{+}$or $\mathrm{Mg}^{2+}$, as expected ${ }^{21,50}$ (Supplementary Fig. 1c). Mutations of key aromatic residues, Phe16 and Trp37 (Supplementary Fig. 1d-e) demonstrated that ssDNA condensation not only depend on phosphate backbone neutralization but also on base capture by the ZF domain. The apo-protein SSHS NC mutant ${ }^{30}$ promoted DNA aggregation without acceleration of DNA mobility as expected for polycationinduced NA aggregation ${ }^{51}$. Finally, Ala substitution of basic residues in the N-terminal domain and the linker demonstrated these residues to be essential for ssDNA condensation, as expected. Strand circularity, e.g. the ssDNA topological constraint, favored intramolecular ssDNA bridging, whereas intermolecular ssDNA-NC-ssDNA interactions were enhanced with linear M13 ssDNA or MS2 RNA (Supplementary Fig. 1f), which demonstrated protein-NA networks involving NAprotein-NA and protein-NA-protein interactions, as proposed previously ${ }^{52}$.

Binding of NCp9 yielded fast-migrating NP condensates for the lowest protein concentrations (Fig. 1c), indistinguishable by AFM from those formed with NCp7 (Supplementary Fig. 2f). However, NCp9-driven NA condensation was seen dramatically associated with a huge fusion process by EMSA (Fig. 1c), DLS (Fig. 2i) and AFM (Fig. 1d, 
Supplementary Fig. 2f-g). Linearity of the ssDNA template resulted in a huge aggregation, demonstrating the presence of an additional NA binding site in SP2 reinforcing NA-NC-NA networks (Fig.2). In contrast to NCp9 or NCp7, reaching a plateau of one NCp15 per 10-12nt, NCp15 binding to any of the three templates yielded NP complexes of lower gel mobility upon protein addition (Fig.1c), similar to canonical ssDNA binding proteins ${ }^{41}$. AFM visualization showed passive ssDNA coating instead of bridging compaction within individual complexes for limiting NCp15 concentration, which then led to globular structures at saturation (Fig.1d, Supplementary Fig. 2h-i). NCp15 and NCp7 retain equivalent net charges ${ }^{14}$ (NCp15 pI 9.93; NCp7 pI 9.59). Therefore, NCp15 binding does not actively compact and aggregate ssNA, confirming previous results ${ }^{5,8}$. With free NCp15, p6 has been proposed to bind to the NC domain ${ }^{8}$. Like NCp15 from HTLV-1 ${ }^{53}$, HIV-1 NCp15 binding to NA might invoke quinary intermolecular p6-NC contacts instead of quinary NA-NC contacts. These interactions may freeze these globular structures and mask or block the NC residues responsible for NA compaction/aggregation. Followed by EMSA, SDS-PAGE and AFM, the dynamics of quinary NC-NA interactions through cleavage of M13 ssDNA-bound NCp15 is verified (Fig. 1e-f) from weak, in the presence of NCp15, to strong with NCp9, and moderate with NCp7. From globular (NCp15), individual NP complexes are progressively converted into intramolecular condensates (NCp7) after an intermediate step of fusion (NCp9).

A superposition of the N-terminal $3_{10}$ helix from the NMR structures of NCp7-SL2 and NCp7-SL3 complexes is shown in Fig. 2a-b and reveals two slightly different NA backbone binding motifs for this domain, which could be virtually sandwiched between two RNA stems, providing a bridge to form RNA-NC-RNA networks ${ }^{52}$. Three additional basic residues over the sixteen present in SP2 poorly explain the dramatic enhancement of the NA gluing capabilities of NCp9. Examination of the NCp9 primary sequence reveals that the NC-SP2 cleavage site surprisingly contains 5 of the 8 residues of the NCp7 SL2-binding motif Lys-Gly-x-Phe-x-x-GlnArg, but oriented in reverse, from C- to N-terminus (Fig. 2a, d). To determine if conformers of this sequence would be compatible with a NA binding site structurally similar to those of the Nterminal $3_{10}$ helix, we performed all-atom molecular dynamics (MD) simulations of an NC-SP2 octapeptide cleavage site. Results of the simulations showed a large conformational area corresponding to a predominantly disordered peptide, similar to other disordered peptide regions in HIV-1 ${ }^{54,55}$. However, three conformer populations were found to lie within $2 \AA$ to $1 \AA$ RMSD with respect to the N-terminal $3_{10}$ helix (Fig. 2c-e), as expected. The K3A/F6A/Q9A-mutation in NCp7 mostly abrogated ssNA aggregation but maintained ssDNA M13 condensation, suggesting this triad to be mostly involved in quinary interactions stabilizing NA:NC networks (Fig. $2 \mathrm{f}-\mathrm{h}$ ). A DLS analysis in low magnesium finally demonstrated a NCp7/NCp9-driven compaction of M13 ssDNA from $100 \mathrm{~nm}$ to $70 \mathrm{~nm}$, followed by a massive fusion/aggregation of these complexes (Fig. 2i). In contrast, the $\mathrm{K} 3 \mathrm{~A} / \mathrm{F} 6 \mathrm{~A} / \mathrm{Q} 9 \mathrm{~A} \mathrm{NCp} 7$ mutant was strongly defective in the 
fusion/aggregation process. Altogether, these data strongly support a model where the Lys(3/59)Gly(4/58)-x-Phe(6/56)-x-x Gln(9/53)-Arg(10/52) octad would act in both NCp7 and NCp9 as a quinary interaction module, establishing bridges between NC-NA complexes at NA saturation (Fig.6a). These positions are highly conserved amongst all the HIV-1 subtypes, except at position 3 where the conservative $\mathrm{K}$ and $\mathrm{R}$ residues are found equiprobable.

Followed by SDS-PAGE under conditions optimized for peptide quantification (Supplementary Fig. 3a-b), in vitro processing of the C- and N-terminal extremities of the NC domain in an environment unfavorable for PR dimers $(0.1 \mathrm{M} \mathrm{NaCl}, \mathrm{pH} 6.25)$ reveals a dramatic acceleration of NCp15, NCp9 and NCp7 production in the presence of ssNA templates (Fig.3a, Supplementary Fig. 3c-e). 100\% of ssDNA- or RNA-bound NCp15 were cleaved in two distinct steps producing $\mathrm{NCp} 9$ and then $\mathrm{NCp} 7$ within minutes, confirming a distributive reaction without consecutive cuts upon the same NCp15 copy (Supplementary Fig. 3c). Without NA, complete $\mathrm{NCp} 15$ cleavage occurred but at a slower rate, only under acidic $(\mathrm{pH} 5.0)$ and high salt (1.5M $\mathrm{NaCl}$ ) conditions (Supplementary Fig. 3d), also concomitantly producing a shorter product (NCp7*). Similar effects were observed with NA for NCp9 cleavage (Supplementary Fig. 3e) and the NC-SP2 cleavage appeared 2-3 times slower than that of SP2-p6 either starting from NCp15 or NCp9, but was completed in minutes, much faster than previously shown ${ }^{16}$. MS2 RNA activation also occurs for the SP1-NC site of a Gag $\Delta$ MA protein (CA-SP1-NC-SP2-p6), confirming recent results using a Gag $\Delta$ p6 (MA-CA-SP1-NC-SP2) protein ${ }^{11}$ (Supplementary Fig. $3 f)$.

We focused on the NC-ssNA NP assemblies and their influence on NCp15 cleavage at pH 6.25 and $0.1 \mathrm{M} \mathrm{NaCl}$. We first compared the influence of large-scale assembly of NCp15 on M13 ssDNA or MS2 RNA versus stoichiometric complexes formed between NC and a TAR RNA stem-loop (Supplementary Fig. 4a). The NA concentrations were varied for a fixed concentration of NCp15. A biphasic effect was observed in presence of either long ssNA, PR reaching maximal activity when NCp15 saturated the ssNA lattices (Supplementary Fig. 4b). A substantial reduction in PR efficacy was observed upon dispersion of NCp15 over the lattice, even though cleavage was maintained at a much higher level than in absence of NA. In contrast, neither a biphasic effect nor a rapid rate was observed in presence of the TAR-RNA. This NA chain-length effect was next followed for NCp15 cleavage, maintaining equal nt concentration for various HIV-1 RNA stemloops and fragments from 61 to 615 nt (Fig. 3b, Supplementary Fig. 4c). A weak NC substrate, $\mathrm{d}(\mathrm{A})_{13}$ oligonucleotide, was ineffective in stimulating PR activity, whereas the TAR and SL3 RNA led to significant, but incomplete stimulation of the reaction. Above a critical threshold ( $\sim 50 \mathrm{nt}-$ length) and for a pH optimum around 6.3 (Supplementary Fig. 4e-f), PR activity scaled nonlinearly with RNA length irrespective of biological origin (Fig. 3b). Without NA, a 5-minute incubation between $\mathrm{NCp} 15$ and $\mathrm{PR}$ in $0.1 \mathrm{M} \mathrm{NaCl}$ at pH 6.25 resulted in no cleavage, while the 
addition of MS2 RNA immediately boosted the reaction (Supplementary Fig. 4d). Diluting PR for fixed NC:PR (10:1) and NA:NC (20nt.:1) ratios revealed a process resistant to dilution for NA larger than $615 \mathrm{nt}$ ( $\mathrm{T}_{12}$ from 0.15 to 1 minute), whereas a strong rate decrease was observed for TAR or cTAR structures ( $T_{12}$ extrapolated to $3 \mathrm{~h}$ when considering the first quarter of the reaction; (Supplementary Fig. 4e). A regular decrease at pH 5.0 and $1.5 \mathrm{M} \mathrm{NaCl}$ was observed in absence of NA ( $T_{12}$ from 4 to $25 \mathrm{~min}$ ). These large NA chains greatly stimulated NCp15 cleavage at 0.1 M salt, with a remarkable pH optimum between 6.0 and 6.5 (Supplementary Fig. 4f). The NCp7* extra-cleavage, previously described, corresponds to a site at position 49-50 as a result of ZF destabilization at low $\mathrm{pH}^{56}$. This product was examined in two $\mathrm{NCp} 15$ cleavage mutants (Supplementary Fig. 4g) and was due to the cumulative effects of both MS2 RNA and acidic pH, which clearly "overcut" the $\mathrm{NC}$ domain at $\mathrm{pH}$ 5.4. A mildly acidic $\mathrm{pH}$ appears therefore beneficial in reducing irregular cleavages of $\mathrm{NCp} 7$ upon high PR turnover. With such optimized conditions that satisfy both PR efficiency and NC folding while restricting NCp7 cryptic site cleavages, we confirmed the RNA length effect for the NCp9-to-NCp7 reaction (Supplementary Fig. 4h-i), leading to a maximal observed rate close to the $\mathrm{T}_{1 / 2}$-value of NCp15-to-NCp9 reaction rate, under conditions where NCp9 and NA appeared strongly aggregated (see Fig. 1). In conclusion, in presence of 1:1 NC ligands (TAR, cTAR, SL3), bound NCp15 appears almost individually distributed in the reaction mix and allow a reaction-diffusion mechanism that accelerate PR turnover but under conditions where native PR is much less stable. Any substance able to increase the local concentration of either the substrate or the enzyme, or both, drives the reaction in the forward direction enhancing enzyme turnover. As such, ssNA length-dependent activation engages a NC crowding effect with NCp15 molecules coating the NA lattice and forming clusters trapping PR independently of the NP complex concentration. These NA-scaffolded clusters allow faster PR turnover, making both SP2-p6 and NC-SP2 cleavages much more efficient. In other words, the NA gluing capabilities of the NC domain induce an RNA-driven sequestration effect on PR.

To better understand this sequestration phenomenon, we used a FRET-based assay that measures the cleavage rate of a MA-CA octapeptide probe in presence of NA and/or NCp15. The assay firstly confirmed the reduction of $\mathrm{PR}$ activity upon $\mathrm{pH}$ increase and salt dilution (Supplementary Fig. 5a-b). The M13 ssDNA appeared as an effective substitute to high salt and boosted PR activity by a factor of 10 at $\mathrm{pH} 5.0$, three times greater than in presence of $1.5 \mathrm{M}$ $\mathrm{NaCl}$. A similar effect also occurred at $\mathrm{pH}$ 6.25, although PR activity was strongly attenuated. These results confirmed that non-specific PR-NA interactions result in enzyme activation ${ }^{12}$. Adding an equimolar amount of $\mathrm{NCp} 15$ at $\mathrm{pH} 5.5$ did not affect the reaction. In contrast, addition of NCp15 bound to ssDNA resulted in a total inhibition of the octapeptide cleavage for $\sim 5 \mathrm{~min}$, before reaching a velocity analogous to that measured in presence of ssDNA alone (Fig. 3d, 
Supplementary Fig. 5c). PR is thus sequestered into the NP complex and completes NCp15 processing prior to cleaving the MA-CA peptide at a rate comparable with ssDNA alone, the delay time being directly proportional to NCp15 concentration with a fixed NCp15:ssDNA ratio (Fig. 3d). These data were interpreted by devising a two-substrate model of NA-modulated enzyme kinetics (Supplementary Note), which partitions the reaction volume between distinct regions that are either occupied (pervaded) or unoccupied (un-pervaded) by NA (Fig. 4a). Reacting species (S1: MA-CA, S2: NCp15, E: PR) exhibit equilibrium absorption $\left(\mathrm{K}_{\mathrm{S} 1}, \mathrm{~K}_{\mathrm{S} 2}\right.$ and $\left.\mathrm{K}_{\mathrm{E}}\right)$ between these regions due to nonsequence-specific NA-binding. In our model, the enzyme escape rate depends on RNP contiguity - the contiguous number $\left(\mathrm{c}=[\mathrm{NCp} 15] \mathrm{n}_{1} /[\mathrm{nt}]\right)$ of $\mathrm{NCp} 15$ molecules bound per NA and not just chain length or NC-NA loading ratio alone. The phase transition is consistent with a minimum critical contiguity threshold $\left(\mathrm{c}_{\text {crit }}\right)$ required to alter enzyme escape rate. Contiguity is still length dependent: fitting a reduced single-substrate model onto the experimental data (Fig. $4 \mathrm{~b}$ ) yields non-linear dependence of $\mathrm{K}_{\mathrm{E}}$ on contiguity with exponent $\xi \sim 0.4$. and $\mathrm{c}_{\text {crit }} \sim 3$. By expanding to a two-substrate competitive assay (Supplementary Note) and incorporating the effects on differential enzyme decay (Fig. 4d), our model is fitted to, and is compatible with the observed sequestration effect (Fig. 3c) giving $\xi \sim 1.2$ and $\mathrm{c}_{\text {crit }} \sim 3$. Sequestration is thus due to reduction in the RNP contiguity across the time-course of the reaction - initially the enzyme is absorbed into the RNP $\left(K_{E}>>1\right)$, after significant processing it is expelled $\left(K_{E}<<1\right)$. The model follows NCp15 cleavage directly (Fig. 4c) and when scaled to in virio concentrations of enzyme and substrate as well as increased NA length, predicts a core condensation time of $\sim 5$ min (Fig. 4e). Our model shows that local crowding within the RNP induces cumulative non-linear effects on non-specific enzyme binding. The absorption equilibrium constant itself depends on this local environment, consistent with quinary interactions between PR, RNA and NCp1 $5^{57}$.

In order to approach this process of RNP condensation in virio, we finally compared by TEM the core content of HIV-1 NL4-3 virus particles assembled with P55 ${ }^{\mathrm{Gag}}$ containing uncleavable NC-SP2 or NC-SP2-p6 sites, thus accumulating NCp9 and NCp15 respectively ${ }^{29}$ (Fig. 5a and Supplementary Fig. 6a). More than $90 \%$ of both NCp9- and NCp7-containing viruses display a morphologically well-formed conical capsid encasing an electron-dark spot corresponding to a condensed RNP. In contrast, more than $80 \%$ of the NCp15-containing viruses display electron-dark diffuse cores. This demonstrates that the strong-gluing NCp9 intermediate actively triggers nucleocapsid condensation, thus reducing the occupied volume and facilitating capsid rearrangement. We next imaged plasma membrane-attached particles of HIV-1 virus produced from latently-infected $\mathrm{ACH} 2$ cells. Washing the cell suspension before fixation enriched the proportion of attached particles engaged in budding. In the presence of a PR inhibitor, all membrane-attached particles appeared immature with a typical electron-dense Gag shell and a bottleneck that characterized budding intermediates (Fig. 5b,d). Without inhibitor, most of the 
attached particles exhibited a dark spot and a closed envelope (Fig. 5c-d). Therefore, the maturation step involving strong-gluing NCp9 occurs visibly in a time frame consistent with both the end of budding ${ }^{58-60}$ and our kinetic model: budding and maturation appear temporally coupled.

\section{Conclusion}

We describe in this study HIV-1 nucleocapsid maturation as a dynamic RNA granule processing, involving differential RNA binding activities of the $\mathrm{NC}$ domain that are dependent on processing state. Weak NC-RNA contacts fit with the concept of quinary interactions ${ }^{3}$, providing a biological "glue" that leads to gRNA condensation in the context of an RNA-directed gel phase ${ }^{2}$. We propose that this RNP follows a dynamic weak-strong-moderate (WSM) quinary model resulting in granular phase-separated RNP condensation (Fig. 6) with a distributive threestep processing mechanism in the order of SP1-NC, SP2-p6 and NC-SP2. Each step alters NCRNA gluing strength within the confined gel phase. The variations in gluing the RNA (in vitro condensation plus aggregation) appear therefore directly linked with the number of amino acid residues weakly contacting NA chains, these contacts being severely limited in NCp15 due to p6 interfering with NC-SP2 NA binding ${ }^{8,14}$ and/or competing with the NA for binding to the NC ZF core. We propose that, in addition to the polycationic nature of the $\mathrm{NC}$ domain ${ }^{20,22,24,53}$, two motifs, one in the N-terminal $3_{10}$ helix and the other, an inverted motif in the NC-SP2 junction are responsible for NC-NA-NC and NA-NC-NA networks providing the molecular glue. In the crowded in virio environment at neutral or mildly acidic $\mathrm{pH}$, our model also involves quinary PR sequestration by the RNP, which dramatically enhances the global efficiency of the sequential cleavage.

Our data confirm, first, that RNA-bound NCp15 avoids active RNP condensation within the NCp15-gRNA intermediate assembly. The intrinsically disordered p6 likely directs a quinary RNA-NCp15 network via NC:p6 intermolecular contacts and weakens the quinary RNA-NC interactions. Such assembly is deficient in actively aggregating within the viral core, while it should allow the 60 PR available in the particle to efficiently access the 2400 SP2-p6 cleavage sites and to jump from site to site within close distances (Fig. 6). PR recently was shown to be activated by interacting with RNA ${ }^{12}$. We demonstrate here that fast PR turnover requires RNAclustered NCp15 for the enzyme to be sequestrated, enhancing its local concentration and allowing it to efficiently cleave the SP2-p6 octapeptide sites.

We suggest the mechanistic origin of this sequestration to be the dynamic formation of a network of quinary interactions, derived from multiple, weakly attractive ternary contacts of PR(SP2-p6)-RNA. Whilst the SP2-p6 octapeptide does not directly appear in contact with RNA, the 
exposed PR basic residues proposed to bind to $\mathrm{RNA}^{12}$ should allow this ternary contact, whereas RNA-bound NCp15 should expose its SP2-p6 cleavage site to RNA-bound PR with the NC-SP2 site being inaccessible. One quinary effect would be that progressive cleavage of the SP2-p6 sites within the RNP results in loss of these ternary contacts, driving the change in enzyme absorption equilibrium - thus expelling PR from the RNP. Following cleavage of an SP2-p6 site, another effect may be a directed, rather than diffusive, propagation of PR. Inaccessibility of NC-SP2 implies a discrimination process that favors trans PR transfer to neighboring NCp15 molecules rather than cis sliding along the NCp9 moiety to cleave the RNA-bound NC-SP2 site, even though only 16 aa sequentially separate the two sites. Cleaved p6, already in contact with a neighboring NC domain, likely directs the trans event. Both effects are complementary to each other and consistent with our model.

When NCp9 forms, the NC-SP2 octapeptide site should bind more easily to RNA due to p6 separation, promoting both the PR recruitment for the NC-SP2 cleavage and the RNA condensation in concert with the other critical NC residues. Again a ternary PR-NCp9-RNA complex would be required, as the free $\mathrm{NCp} 9$ appeared here as a poor substrate for PR, confirming previous results ${ }^{16}$. We propose that the NC-SP2 site is in direct contact with RNA by way of the QxxFxxK triad. The critical NC-SP2 octapeptide also binds within the PR internal catalytic site for cleavage: this cleavage should require displacement of the bound RNA upon the surface of PR allowing a quinary contact between RNA and PR. In addition, longer RNA segments are required to strongly enhance NCp9 cleavage suggesting that PR transfers directly from cleaved $\mathrm{NCp} 9$ (i.e. NCp7) to neighboring NCp9. RNA condensation does not seem to provide any critical physical barrier to PR as NCp9 processing appears quite similar whether the reaction starts from NCp15 or NCp9 (Fig. 3a). This suggests a perfect fit for ordered turnover by PR from site to site with two distinct waves, NCp15-to-NCp9 followed by NCp9-to-NCp7.

Quinary interactions undoubtedly offer a missing link between molecular and cellular biology ${ }^{1-4}$. In the HIV-1 model, the granulation dynamics shown here opens new avenues at the mesoscopic scale to better understand how the surrounding capsid progresses towards a conical reassembly and what the implication and relocation of RT and IN proteins are. While both mature during GagPol processing, IN has been shown recently to be a key actor in properly coordinating RNP granular condensation and capsid reassembly within the viral particle ${ }^{61,62}$. These dynamics also offer new schemes to revisit the proposed implications of HIV-1 Nef, Vif, Tat and Vpr auxiliary proteins within the design of an infectious particle ${ }^{63}$. It strongly argues finally for tight control of GagPol incorporation and PR auto-processing supported by the NC domain in GagPol during particle formation ${ }^{64,65}$ : this domain should help to position GagPol in the Gag assembly facilitating the processed PR directed by the gRNA to digest the Gag-domains NC, SP2, p6. As highlighted by an independent study looking for production of GagPol VLPs without any viral 
accessory genes ${ }^{66}$, the concomitant interactions of the exposed $\mathrm{p} 6$ domains with the ESCRT components, ALIX and more especially TSG10127,67,68, tightly coordinate complete processing of Gag within just a few minutes of particle release. This is in accordance with what we show here, namely, the exclusive release and processing of mature HIV-1 particles, including a condensed nucleocapsid, accelerated to the same time frame due to a gel phaseseparated effect.

Finally, we highlight here NC-RNA interactions as highly attractive pharmacological targets during particle production ${ }^{69}$ and we shed light on new approaches for studying RNP assemblies where RNA provides a scaffolding role for crowded architectures, emergent mechanisms and their quinary regulation ${ }^{2,4,70}$.

\section{References}

1 Buchan, J. R. mRNP granules. Assembly, function, and connections with disease. RNA Biol 11, 1019-1030, doi:10.4161/15476286.2014.972208 (2014).

2 Guo, L. \& Shorter, J. It's Raining Liquids: RNA Tunes Viscoelasticity and Dynamics of Membraneless Organelles. Mol Cell 60, 189-192, doi:10.1016/j.molcel.2015.10.006 (2015).

3 Chien, P. \& Gierasch, L. M. Challenges and dreams: physics of weak interactions essential to life. Mol Biol Cell 25, 3474-3477, doi:10.1091/mbc.E14-06-1035 (2014).

4 McConkey, E. H. Molecular evolution, intracellular organization, and the quinary structure of proteins. Proc Natl Acad Sci U S A 79, 3236-3240 (1982).

5 Mirambeau, G. et al. Transmission electron microscopy reveals an optimal HIV-1 nucleocapsid aggregation with single-stranded nucleic acids and the mature HIV-1 nucleocapsid protein. J Mol Biol 364, 496-511, doi:10.1016/j.jmb.2006.08.065 (2006).

6 Mirambeau, G. et al. HIV-1 protease and reverse transcriptase control the architecture of their nucleocapsid partner. PLoS One 2, e669, doi:10.1371/journal.pone.0000669 (2007).

7 Mirambeau, G., Lyonnais, S. \& Gorelick, R. J. Features, processing states, and heterologous protein interactions in the modulation of the retroviral nucleocapsid protein function. RNA Biol 7, 724-734 (2010).

8 Wang, W. et al. Distinct nucleic acid interaction properties of HIV-1 nucleocapsid protein precursor NCp15 explain reduced viral infectivity. Nucleic Acids Res 42, 7145-7159, doi:10.1093/nar/gku335 (2014).

9 Sundquist, W. I. \& Krausslich, H. G. HIV-1 assembly, budding, and maturation. Cold Spring Harb Perspect Med 2, a006924, doi:10.1101/cshperspect.a006924 (2012).

10 Konvalinka, J., Krausslich, H. G. \& Muller, B. Retroviral proteases and their roles in virion maturation. Virology 479-480, 403-417, doi:10.1016/j.virol.2015.03.021 (2015).

11 Deshmukh, L., Ghirlando, R. \& Clore, G. M. Conformation and dynamics of the Gag polyprotein of the human immunodeficiency virus 1 studied by NMR spectroscopy. Proc Natl Acad Sci U S A 112, 3374-3379, doi:10.1073/pnas.1501985112 (2015).

12 Potempa, M. et al. A Direct Interaction with RNA Dramatically Enhances the Catalytic Activity of the HIV-1 Protease In Vitro. J Mol Biol 427, 2360-2378, doi:10.1016/j.jmb.2015.05.007 (2015). 
de Marco, A. et al. Role of the SP2 domain and its proteolytic cleavage in HIV-1 structural maturation and infectivity. J Virol 86, 13708-13716, doi:10.1128/JVI.01704-12 (2012). Wu, T., Gorelick, R. J. \& Levin, J. G. Selection of fully processed HIV-1 nucleocapsid protein is required for optimal nucleic acid chaperone activity in reverse transcription. Virus Res 193, 52-64, doi:10.1016/j.virusres.2014.06.004 (2014). Todd, M. J., Semo, N. \& Freire, E. The structural stability of the HIV-1 protease. J Mol Biol 283, 475-488, doi:10.1006/jmbi.1998.2090 (1998).

16 Pettit, S. C., Sheng, N., Tritch, R., Erickson-Viitanen, S. \& Swanstrom, R. The regulation of sequential processing of HIV-1 Gag by the viral protease. Adv Exp Med Biol 436, 1525 (1998).

17 Sheng, N. et al. Determinants of the human immunodeficiency virus type 1 p15NC-RNA interaction that affect enhanced cleavage by the viral protease. J Virol 71, 5723-5732 (1997).

18 Amarasinghe, G. K. et al. NMR structure of the HIV-1 nucleocapsid protein bound to stem-loop SL2 of the Psi-RNA packaging signal. Implications for genome recognition. $J$ Mol Biol 301, 491-511, doi:DOI 10.1006/jmbi.2000.3979 (2000).

19 De Guzman, R. N. et al. Structure of the HIV-1 nucleocapsid protein bound to the SL3 Psi-RNA recognition element. Science 279, 384-388, doi:DOI 10.1126/science.279.5349.384 (1998).

20 Wu, H. et al. Differential contribution of basic residues to HIV-1 nucleocapsid protein's nucleic acid chaperone function and retroviral replication. Nucleic Acids Res 42, 25252537, doi:10.1093/nar/gkt1227 (2014).

21 Le Cam, E. et al. Properties and growth mechanism of the ordered aggregation of a model RNA by the HIV-1 nucleocapsid protein: an electron microscopy investigation. Biopolymers 45, 217-229 (1998).

22 Khan, R. \& Giedroc, D. P. Nucleic acid binding properties of recombinant Zn2 HIV-1 nucleocapsid protein are modulated by $\mathrm{COOH}$-terminal processing. J Biol Chem 269, 22538-22546 (1994).

23 Wu, H., Rouzina, I. \& Williams, M. C. Single-molecule stretching studies of RNA chaperones. RNA Biol 7, 712-723 (2010).

24 Cruceanu, M. et al. Nucleic acid binding and chaperone properties of HIV-1 Gag and nucleocapsid proteins. Nucleic Acids Res 34, 593-605 (2006).

25 Lee, S. K., Potempa, M. \& Swanstrom, R. The choreography of HIV-1 proteolytic processing and virion assembly. J Biol Chem 287, 40867-40874, doi:10.1074/jbc.R112.399444 (2012).

26 Konnyu, B. et al. Gag-Pol processing during HIV-1 virion maturation: a systems biology approach. PLoS Comput Biol 9, e1003103, doi:10.1371/journal.pcbi.1003103 (2013).

27 Dussupt, V. et al. Basic residues in the nucleocapsid domain of Gag are critical for late events of HIV-1 budding. J Virol 85, 2304-2315, doi:10.1128/JVI.01562-10 (2011).

28 Moore, M. D. et al. Suboptimal inhibition of protease activity in human immunodeficiency virus type 1: effects on virion morphogenesis and RNA maturation. Virology 379, 152-160, doi:10.1016/j.virol.2008.06.030 (2008).

29 Coren, L. V. et al. Mutational analysis of the C-terminal gag cleavage sites in human immunodeficiency virus type 1. J Virol 81, 10047-10054, doi:10.1128/JVI.02496-06 (2007).

30 Guo, J. et al. Zinc finger structures in the human immunodeficiency virus type 1 nucleocapsid protein facilitate efficient minus- and plus-strand transfer. J Virol 74, 89808988 (2000).

31 Stewart-Maynard, K. M. et al. Retroviral nucleocapsid proteins display nonequivalent levels of nucleic acid chaperone activity. J Virol 82, 10129-10142, doi:10.1128/JVI.01169-08 (2008). 

immunodeficiency virus type $1 \mathrm{cDNA}$ ends by purified integrase in vitro: stimulation by the viral nucleocapsid protein. J Virol 73, 6670-6679 (1999). nucleocapsid protein's nucleic acid chaperone activity and retroviral replication. Virus Res 171, 263-277, doi:10.1016/j.virusres.2012.07.008 (2013). HIV-1 proteinase. Biol Chem Hoppe Seyler 371, 265-272 (1990). are successful strategies for inhibiting wild-type and multidrug-resistant mutated HIV-1 proteases. Biochemistry 48, 379-387, doi:10.1021/bi801422u (2009). retroviral protease dimerization and activation. J Virol 77, 3319-3325 (2003).

37 Rothemund, P. W. Folding DNA to create nanoscale shapes and patterns. Nature 440, 297-302, doi:10.1038/nature04586 (2006).

38 Sinck, L. et al. In vitro dimerization of human immunodeficiency virus type 1 (HIV-1) spliced RNAs. RNA 13, 2141-2150, doi:10.1261/rna.678307 (2007).

39 Abd El-Wahab, E. W. et al. Specific recognition of the HIV-1 genomic RNA by the Gag precursor. Nat Commun 5, 4304, doi:10.1038/ncomms5304 (2014).

40 Goldschmidt, V. et al. Structural variability of the initiation complex of HIV-1 reverse transcription. J Biol Chem 279, 35923-35931, doi:10.1074/jbc.M404473200 (2004).

41 Hamon, L. et al. High-resolution AFM imaging of single-stranded DNA-binding (SSB) protein--DNA complexes. Nucleic Acids Res 35, e58, doi:10.1093/nar/gkm147 (2007).

42 Gonda, M. A., Aaronson, S. A., Ellmore, N., Zeve, V. H. \& Nagashima, K. Ultrastructural studies of surface features of human normal and tumor cells in tissue culture by scanning and transmission electron microscopy. J Natl Cancer Inst 56, 245-263 (1976). Ott, D. E. et al. Mutational analysis of the hydrophobic tail of the human immunodeficiency virus type $1 \mathrm{p} 6(\mathrm{Gag})$ protein produces a mutant that fails to package its envelope protein. J Virol 73, 19-28 (1999).

44 Clouse, K. A. et al. Monokine regulation of human immunodeficiency virus-1 expression in a chronically infected human T cell clone. J Immunol 142, 431-438 (1989).

45 Lopez-Iglesias, C. \& Puvion-Dutilleul, F. Visualization of glycoproteins after tunicamycin and monensin treatment of herpes simplex virus infected cells. J Ultrastruct Mol Struct Res 101, 75-91 (1988).

46 Sadiq, S. K. \& Coveney, P. V. Computing the role of near attack conformations in an enzyme-catalyzed nucleophilic bimolecular reaction. Journal of chemical theory and computation 11, 316-324, doi:10.1021/ct5008845 (2015).

47 Prabu-Jeyabalan, M., Nalivaika, E. A., King, N. M. \& Schiffer, C. A. Structural basis for coevolution of a human immunodeficiency virus type 1 nucleocapsid-p1 cleavage site with a V82A drug-resistant mutation in viral protease. J Virol 78, 12446-12454, doi:10.1128/JVI.78.22.12446-12454.2004 (2004).

48 Duan, Y. et al. A point-charge force field for molecular mechanics simulations of proteins based on condensed-phase quantum mechanical calculations. J Comput Chem 24, 19992012, doi:10.1002/jcc.10349 (2003).

49 Harvey, M. J., Giupponi, G. \& Fabritiis, G. D. ACEMD: Accelerating Biomolecular Dynamics in the Microsecond Time Scale. Journal of chemical theory and computation 5, 1632-1639, doi:10.1021/ct9000685 (2009).

50 Vo, M. N., Barany, G., Rouzina, I. \& Musier-Forsyth, K. Effect of $\mathrm{Mg}(2+)$ and $\mathrm{Na}(+)$ on the nucleic acid chaperone activity of HIV-1 nucleocapsid protein: implications for reverse transcription. J Mol Biol 386, 773-788, doi:10.1016/j.jmb.2008.12.073 (2009). 

nucleocapsid protein revealed by single molecule stretching. Proc Natl Acad Sci U S A 98, 6121-6126, doi:10.1073/pnas.101033198 (2001).

52 Fisher, R. J. et al. Complex interactions of HIV-1 nucleocapsid protein with oligonucleotides. Nucleic Acids Res 34, 472-484, doi:10.1093/nar/gkj442 (2006).

Qualley, D. F. et al. C-terminal domain modulates the nucleic acid chaperone activity of human T-cell leukemia virus type 1 nucleocapsid protein via an electrostatic mechanism. J Biol Chem 285, 295-307, doi:10.1074/jbc.M109.051334 (2010). competitive heteropolymer cleavage. J Phys Chem B 115, 11017-11027, doi:10.1021/jp206321b (2011). Fluctuations of Disordered HIV-1 Fusion Peptide in Solution. Journal of chemical theory and computation 9, 2870-2874, doi:10.1021/ct300856r (2013).

Roberts, M. M., Copeland, T. D. \& Oroszlan, S. In situ processing of a retroviral nucleocapsid protein by the viral proteinase. Protein Eng 4, 695-700 (1991).

57 Monteith, W. B., Cohen, R. D., Smith, A. E., Guzman-Cisneros, E. \& Pielak, G. J. Quinary structure modulates protein stability in cells. Proc Natl Acad Sci U S A 112, 1739-1742, doi:10.1073/pnas.1417415112 (2015). Jouvenet, N., Simon, S. M. \& Bieniasz, P. D. Visualizing HIV-1 assembly. J Mol Biol 410, 501-511, doi:10.1016/j.jmb.2011.04.062 (2011).

59 Votteler, J. \& Sundquist, W. I. Virus budding and the ESCRT pathway. Cell Host Microbe 14, 232-241, doi:10.1016/j.chom.2013.08.012 (2013).

60 Prescher, J. et al. Super-resolution imaging of ESCRT-proteins at HIV-1 assembly sites. PLoS Pathog 11, e1004677, doi:10.1371/journal.ppat.1004677 (2015).

61 Fontana, J. et al. Distribution and Redistribution of HIV-1 Nucleocapsid Protein in Immature, Mature, and Integrase-Inhibited Virions: a Role for Integrase in Maturation. J Virol 89, 9765-9780, doi:10.1128/JVI.01522-15 (2015). Kessl, J. J. et al. HIV-1 Integrase Binds the Viral RNA Genome and Is Essential during Virion Morphogenesis. Cell 166, 1257-1268, doi:DOI 10.1016/j.cell.2016.07.044 (2016). Cuccurullo, E. C., Valentini, C. \& Pizzato, M. Retroviral factors promoting infectivity. Prog Mol Biol Trans/ Sci 129, 213-251, doi:10.1016/bs.pmbts.2014.10.008 (2015). Cen, S. et al. Incorporation of pol into human immunodeficiency virus type $1 \mathrm{Gag}$ viruslike particles occurs independently of the upstream Gag domain in Gag-pol. J Virol 78, 1042-1049 (2004).

65 Figueiredo, A. et al. Potent nonnucleoside reverse transcriptase inhibitors target HIV-1 Gag-Pol. PLoS Pathog 2, e119, doi:10.1371/journal.ppat.0020119 (2006).

66 Bendjennat, M. \& Saffarian, S. The Race against Protease Activation Defines the Role of ESCRTs in HIV Budding. PLoS Pathog 12, e1005657, doi:10.1371/journal.ppat.1005657 (2016).

67 Chamontin, C. et al. HIV-1 nucleocapsid and ESCRT-component Tsg101 interplay prevents HIV from turning into a DNA-containing virus. Nucleic Acids Res 43, 336-347, doi:DOI 10.1093/nar/gku1232 (2015).

68 Popova, E., Popov, S. \& Gottlinger, H. G. Human immunodeficiency virus type 1 nucleocapsid p1 confers ESCRT pathway dependence. J Virol 84, 6590-6597, doi:DOI 10.1128/JVI.00035-10 (2010).

69 Mori, M. et al. Nucleocapsid Protein: A Desirable Target for Future Therapies Against HIV-1. Curr Top Microbiol Immunol 389, 53-92, doi:10.1007/82_2015_433 (2015).

70 Dumont, S. \& Prakash, M. Emergent mechanics of biological structures. Mol Biol Cell 25, 3461-3465, doi:DOI 10.1091/mbc.E14-03-0784 (2014). 
Acknowledgements. We thank Carlo Carolis, Biomolecular Screening and Protein Technology Unit (CRG), Carmen Lopez Iglesias and the TEM-SEM facilities of Science and Technical Centers of the Universitat de Barcelona (CCiT-UB), Cathy V. Hixson and Donald G. Johnson of Leidos Biomedical Research, Inc. and Eric Le Cam (IGR and CNRS). This work was supported in part by the European Project THINPAD "Targeting the HIV-1 Nucleocapsid Protein to fight Antiretroviral Drug Resistance" (FP7-Grant Agreement 601969), by Foundation Clinic, by ANRS, by SIDACTION, and with Federal funds from the NCI/NIH, under Contract No. HHSN261200800001E with Leidos Biomedical Research, Inc. (R.J.G.). S.L. acknowledges funding by the Marie-Curie IEF fellowship (FP7-Grant Agreement 237738) and is grateful to Maria Solà (IBMB-CSIC). S.K.S. and A.M. acknowledge support from amfAR Mathilde Krim Fellowship in Basic Biomedical Research number 108680 and the Spanish Ministry of Economy and Competitiveness and FEDER (Grant no. SAF2013-46077-R). S.K.S. also gratefully acknowledges support from the Volkswagen Foundation "Experiment! Funding Initiative" grant number 93874 and from the Klaus Tschira Stiftung.

Author Contributions. S. L. and G. M. conceived and designed the experiments. S. L. and T. E. performed the DNA mobility shift assays. S. L. did the AFM experiments. J. O. and S. L. did the DLS experiments. L. D., T. E., X. T., M. O.-O., M. R.-R. did the protease experiments. S.K. S. conceived and developed the theory, designed and implemented the kinetic model and the molecular dynamics simulations. R. G. and C. T. prepared NC proteins. L. D. and M. R.-R. prepared PR proteins. J.-C. P. and R. M. provided the collection of HIV-1 RNA fragments. R. G., C. O.-L. and N. G. did the virological and TEM experiments, with J. M. G. and A. M. advising. S. L., S.K. S. and G. M. analyzed the data and wrote the manuscript with strong support of R.G. and input from J. M. G., A. M., J.-C. P., R. M. and C. T. Figures were developed and arranged by S. L., G. M. and S. K. S. S.L. realized the illustrations.

Supplementary Information is available with this article. The authors declare no competing financial interests. Readers are welcome to comment on the online version of the article. Correspondence and requests for materials and modelling data should be addressed to S.L. (sebastien.lyonnais@cemipai.cnrs.fr), $\quad$ S.K.S. $\quad$ (kashif.sadiq@h-its.org) or $\quad$ G.M. (gilles.mirambeau@upmc.fr). 


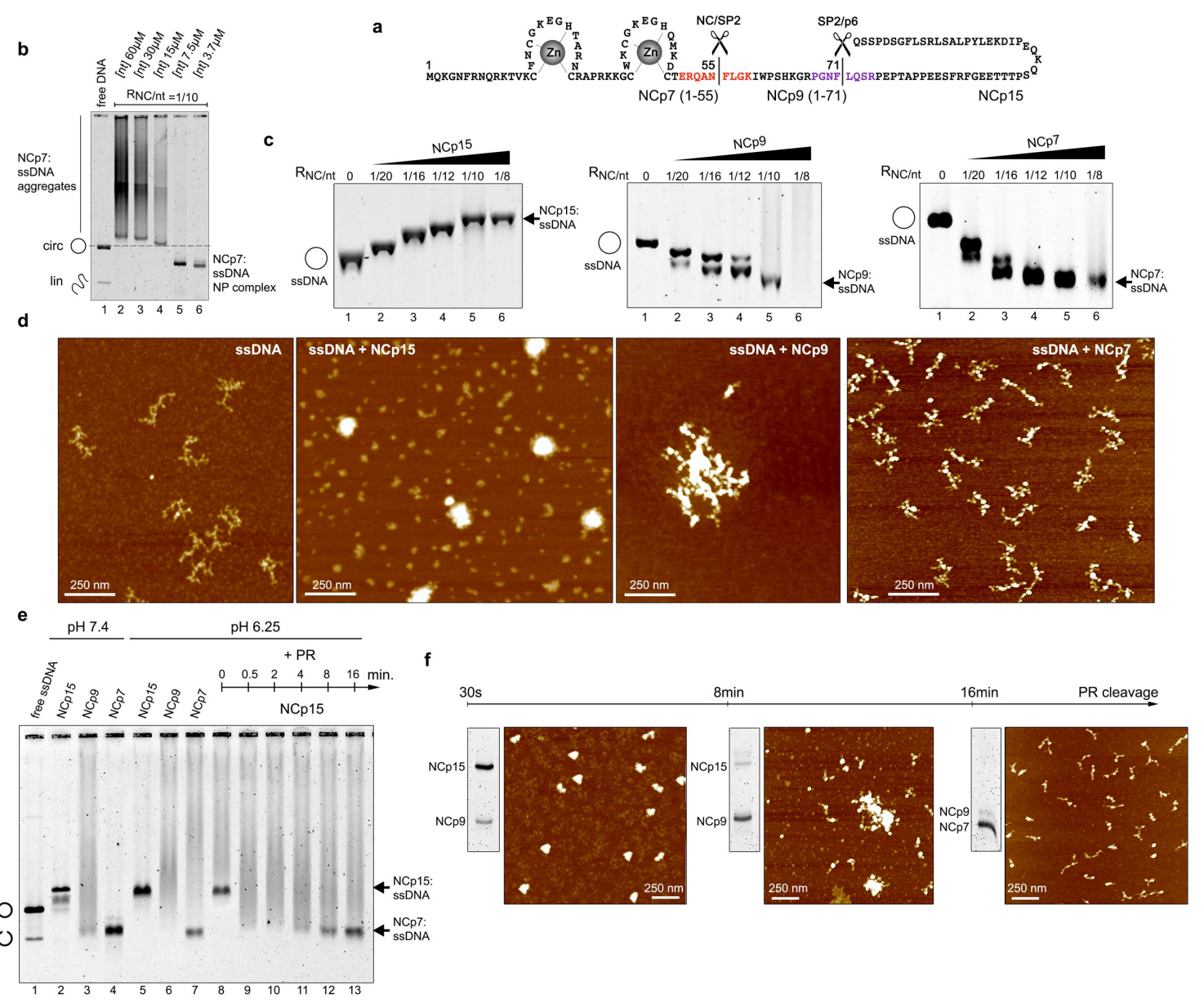

Figure 1 | WSM-gluing properties of NCp15, NCp9 and NCp7. a, Sequence of NCp15 and PR cleavage sites. b, on agarose gel, the electrophoretic migration of M13 ssDNA:NCp7 NP complexes (1NCp7/10nt) for serial dilutions of both DNA (expressed in total [nt]) and NCp7 shows a switch from aggregation to intramolecular condensation. Position of the unbound circular (circ) ssDNA and trace of linear (lin) molecules is indicated on the left. c, M13 ssDNA:NCp15 complexes are up-shifted by EMSA upon increasing protein concentration, while ssDNA:NCp9 and ssDNA:NCp7 complexes are accelerated, thus compacted, and aggregate after NA saturation. $\mathrm{R}_{\mathrm{NC} / \mathrm{nt}}$ indicate the protein/nucleotide ratio. d, Condensation of M13 ssDNA by NCp7 and NCp9 is visualized by AFM and show intramolecular condensates involving DNA strand bridging. NCp9 aggregate these condensates while ssDNA:NCp15 complexes appear globular. The cleavage kinetics of M13 ssDNA-bound NCp15 by PR was followed by EMSA (e), SDS-PAGE and AFM (f), showing weak-strong-moderate gluing properties of NCp15, NCp9 and NCp7, respectively, along the sequential maturation of NCp7 by PR. In the EMSA gel are shown NP complexes migration controls at $\mathrm{pH} 7.4$ and $\mathrm{pH} 6.25$ (optimal for PR cleavage). 


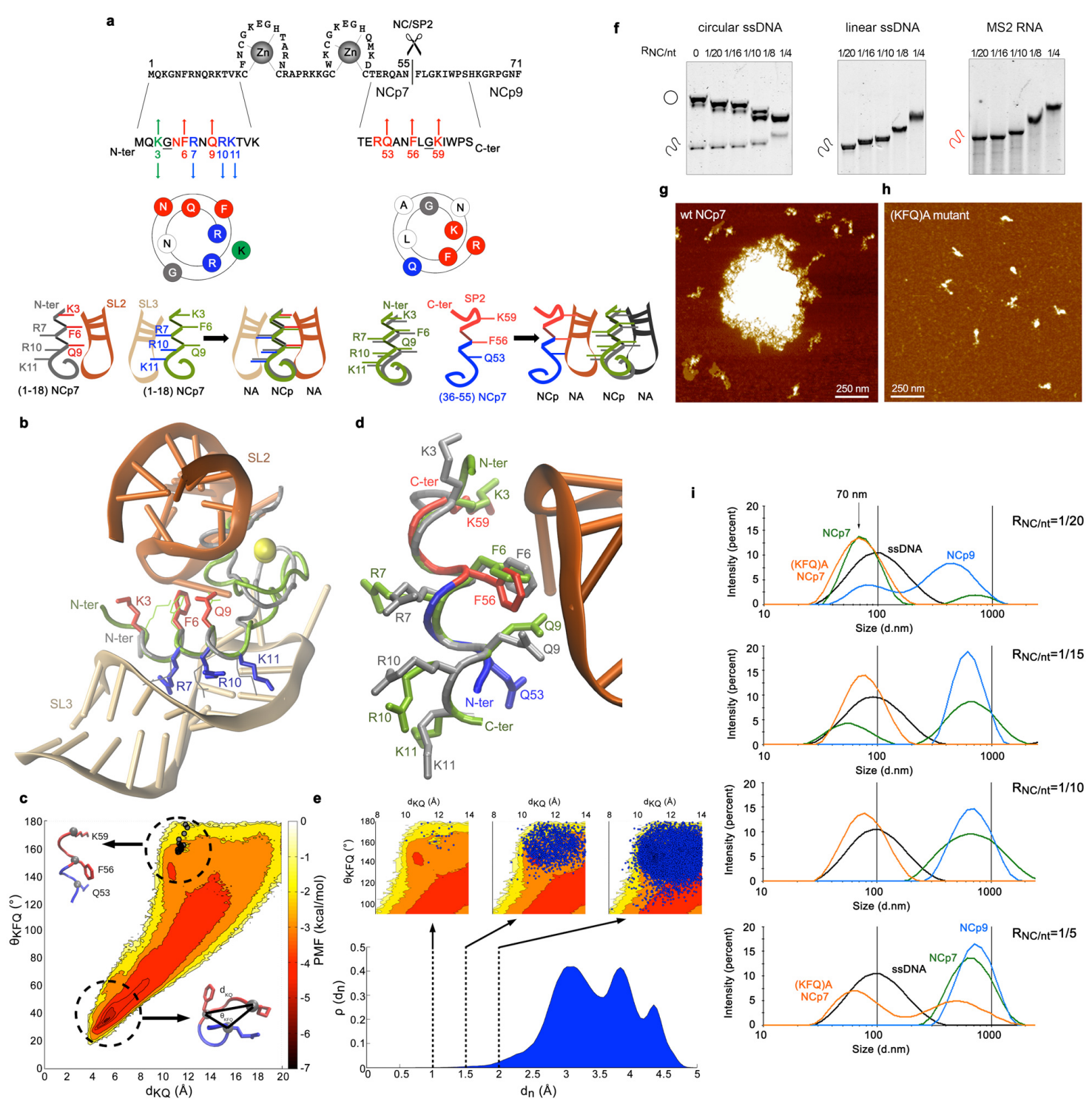

Figure 2 NC:NA quinary interactions. a, The N-terminal $3_{10}$ helix and the NC-SP2 cleavage site contain DNA strand bridging motifs. The Wenxiang diagram shows the residues of the N-terminal $3_{10}$ helix in contact with SL3-RNA (red) or SL2-RNA (blue). The diagram of the 53-60 sequence shows the Q53-F56-G58-K59 motif mirroring the K3-G4-F6-Q9 motif in contact with SL2. The schematics indicate possible NA-NC-NA network assemblies provided by such NA binding domains. b, Superposition of NC-SL3 (PDB ID: 1A1T) and NC-SL2 (PDB ID: 1F6U) N-terminal domain in complex with their respective NA ligands. c, The NC-SP2 apo-octapeptide exhibits substantial flexibility and is free-energetically dominated by a turn-like structure with $\mathrm{d}_{\mathrm{KQ}} \sim 4-6 \AA$ structure $\theta_{\mathrm{KFQ}} \sim 20^{\circ}-40^{\circ}$ yielding a PMF of 5-7 kcal $/ \mathrm{mol}$. The apo-ensemble conformers sample a region with $\mathrm{d}_{\mathrm{KQ}} \sim 10-12 \AA$ with $\theta_{\mathrm{KFQ}}$ $\sim 140^{\circ}-180^{\circ}$ with less frequency (PMF 1-3 kcal $/ \mathrm{mol}$ ) where fit all NMR conformers of $1 \mathrm{~F} 6 \mathrm{U}$, thus compatible with a $3{ }_{10}$ helical structure. d, Illustration of best-fitting structural conformer 
bioRxiv preprint first posted online Jan. 26,2019 ; doi: http://dx.doi.org/10.1101/528638. The copyright holder for this preprint (which was not peer-reviewed) is the author/funder, who has granted bioRxiv a license to display the preprint in perpetuity. All rights reserved. No reuse allowed without permission.

of the 52-59 segment superimposed head-to-tail with the N-terminal domain of 1F6U. e, Three conformers populations were found to lie within $2 \AA \operatorname{RMSD}\left(9.1 \times 10^{-5} \%\right), 1.5 \AA\left(1.3 \times 10^{-5} \%\right.$ ) and $1 \AA\left(3 \times 10^{-7} \%\right)$ with respect to the $\mathrm{N}$-terminal $3_{10}$ helix. Mapping the conformers onto the $\mathrm{d}_{\mathrm{KQ}}-\theta_{\mathrm{KFQ}}$ order parameters (blue circles) show they occupy the same region of the conformational sub-space. f, K3AF6AQ9A mutations in NCp7 strongly reduced the protein's capability to aggregate circular and linear M13 ssDNA or MS2 RNA (compare with Fig. 1c, NCp7). g-h In the absence of magnesium, wt-NCp7 (1NCp7/10nt) strongly aggregates M13 ssDNA whereas the K3AF6AQ9A mutant preferentially condenses the ssDNA. i, By DLS in the absence of magnesium, M13 ssDNA (black) is condensed by the K3AF6AQ9A NCp7 mutant (orange), condensed and next aggregated by $\mathrm{NCp} 7$ (green) or aggregated by $\mathrm{NCp} 9$ (blue). 
bioRxiv preprint first posted online Jan. 26, 2019; doi: http://dx.doi.org/10.1101/528638. The copyright holder for this preprint (which was not peer-reviewed) is the author/funder, who has granted bioRxiv a license to display the preprint in perpetuity. All rights reserved. No reuse allowed without permission.
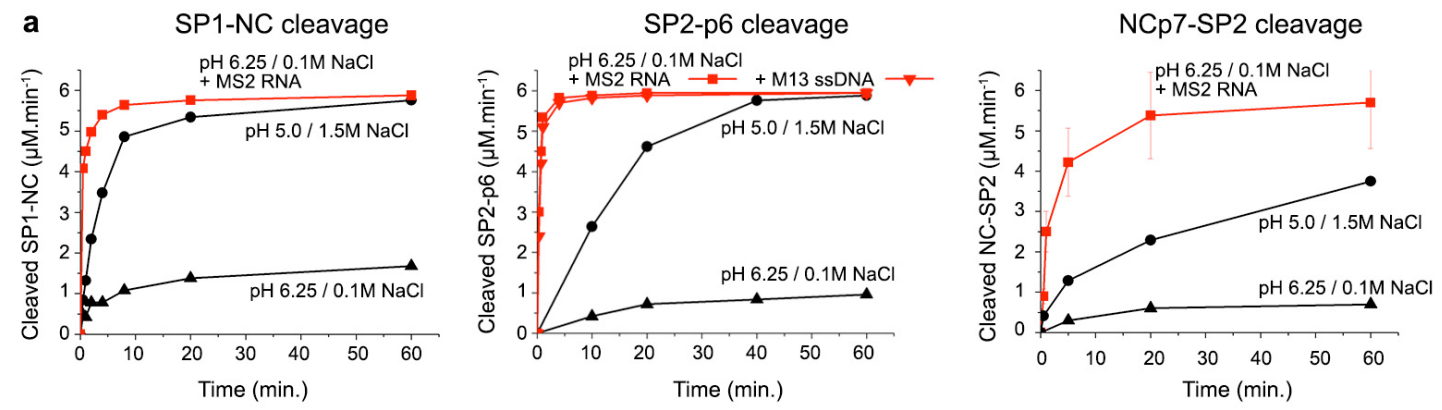

b

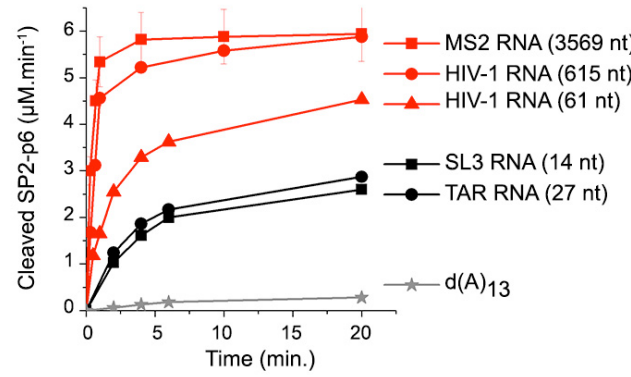

C
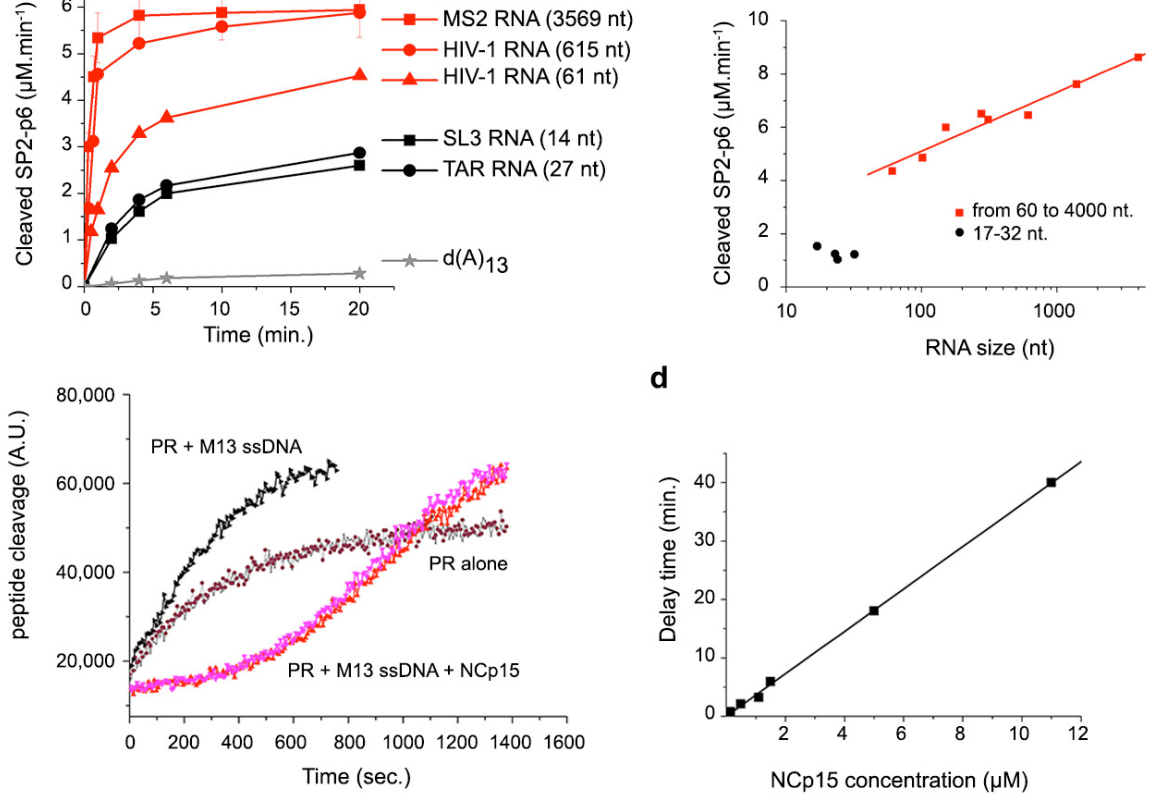

Figure 3 | Nucleocapsid maturation is promoted by NA through sequestration of PR. a, NC binding to M13 ssDNA or MS2 RNA strongly activates in vitro cleavage of SP1-NC, SP2-p6 and NCp7-SP2 sites by PR under unfavourable conditions (pH 6.25 and $0.1 \mathrm{M} \mathrm{NaCl}$; see Supplementary Fig. 3a-b for assay details). b, In vitro cleavage of NCp15 by PR is activated in a ssNA length-dependent manner. c, Cleavage of a MA-CA probe by PR is activated by ssDNA and is delayed in presence of ssDNA:NCp15 complexes as the latter sequester PR. MA-CA proteolysis was followed by FRET. d, Delay of the FRET probe cleavage extrapolated from (c) as a function of M13 ssDNA:NCp15 complex concentration $\left(\mathrm{R}_{\mathrm{NCp} 15 / \mathrm{nt}}=1 / 20\right)$. 
a
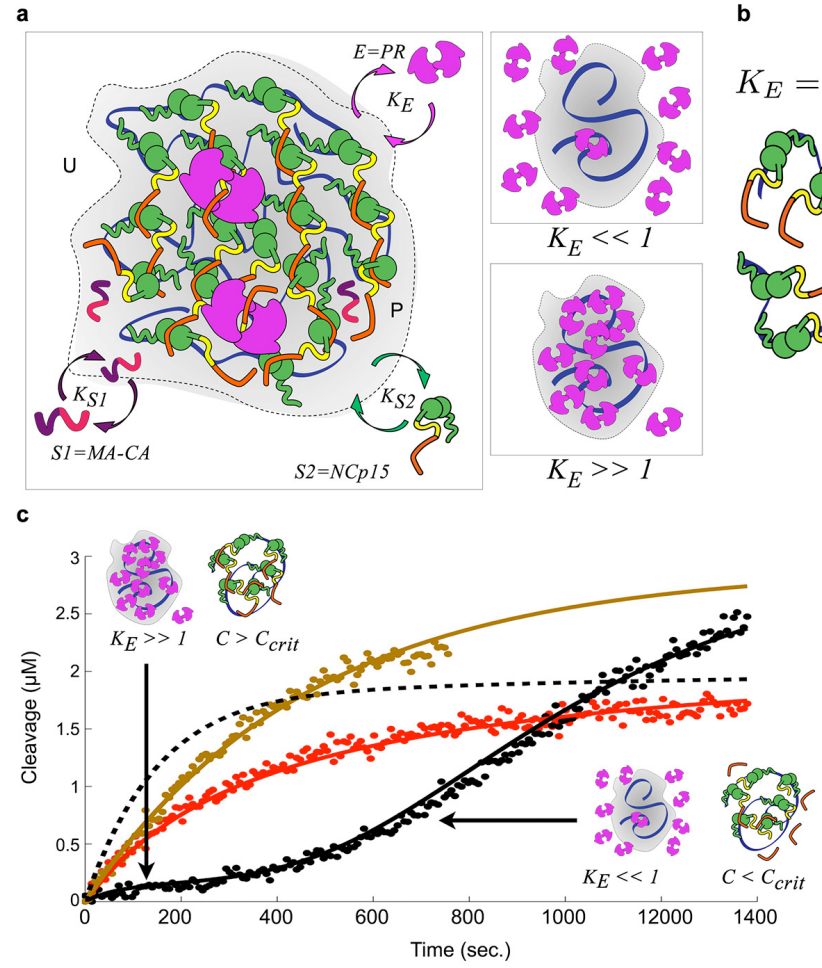

b

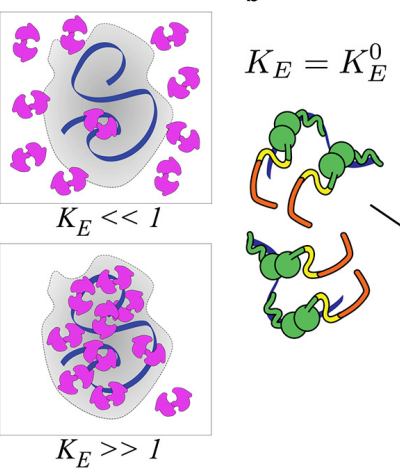

d
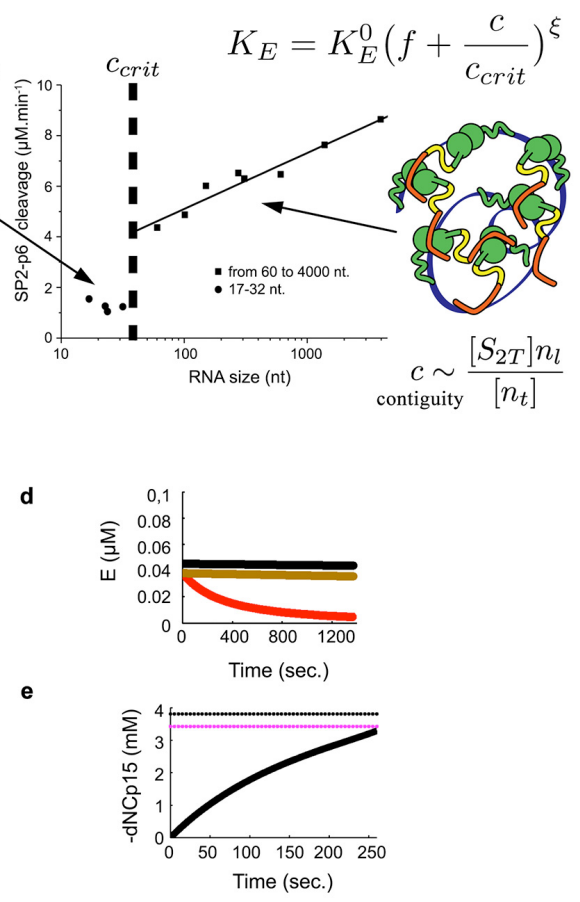

Figure 4 | Kinetic model of two-substrate (S1, S2) processing by an enzyme (E) in a RNP. a, Reaction rate is governed by a combination of effective concentration in a volume domain and absorption kinetics for different species. For $\mathrm{K}_{\mathrm{E}}>>1$, $\mathrm{PR}$ is sequestered into the RNP-pervaded volume whilst for $\mathrm{K}_{\mathrm{E}}<<1$ it is forced out. $\mathbf{b}$, A one-substrate model is fit to experimental data to determine non-linear (exponent $\xi$ ) $\mathrm{K}_{\mathrm{E}}$ dependence on the contiguous number $c$ of S2 molecules bound per NA above a critical threshold, $c_{c r i t}$ c , Fitted two-substrate model of sequestration (black) with competitive substrate alone (red) NA-present competitive substrate (brown) data. The early reaction is dominated by high contiguity $\left(c>c_{c r i t}, \mathrm{~K}_{\mathrm{E}}>>1\right)$ inducing enzyme sequestration. This effect dissipates upon processing $\left(c<c_{c r i t}, \mathrm{~K}_{\mathrm{E}}<<1\right) .90 \%$ of NCp15 cleavage (dashed line) occurs within 400 s. d, Differential enzyme decay: NA stabilizes the PR dimer; the NA-absent reaction decays with experimental half-life. e, Scaling to in virio conditions yields 90\% NCp15 processing (purple) within $260 \mathrm{~s}$. 
bioRxiv preprint first posted online Jan. 26, 2019; doi: http://dx.doi.org/10.1101/528638. The copyright holder for this preprint (which was not peer-reviewed) is the author/funder, who has granted bioRxiv a license to display the preprint in perpetuity. All rights reserved. No reuse allowed without permission.
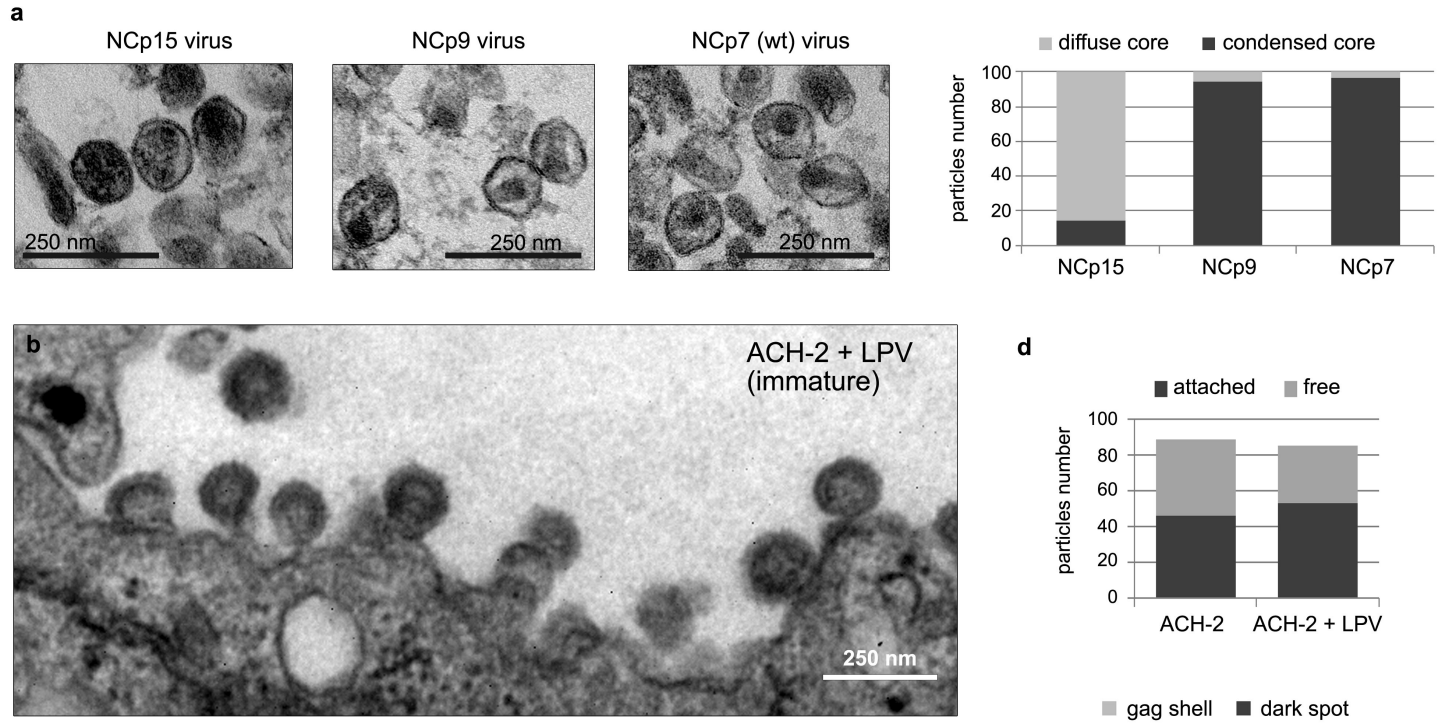

d
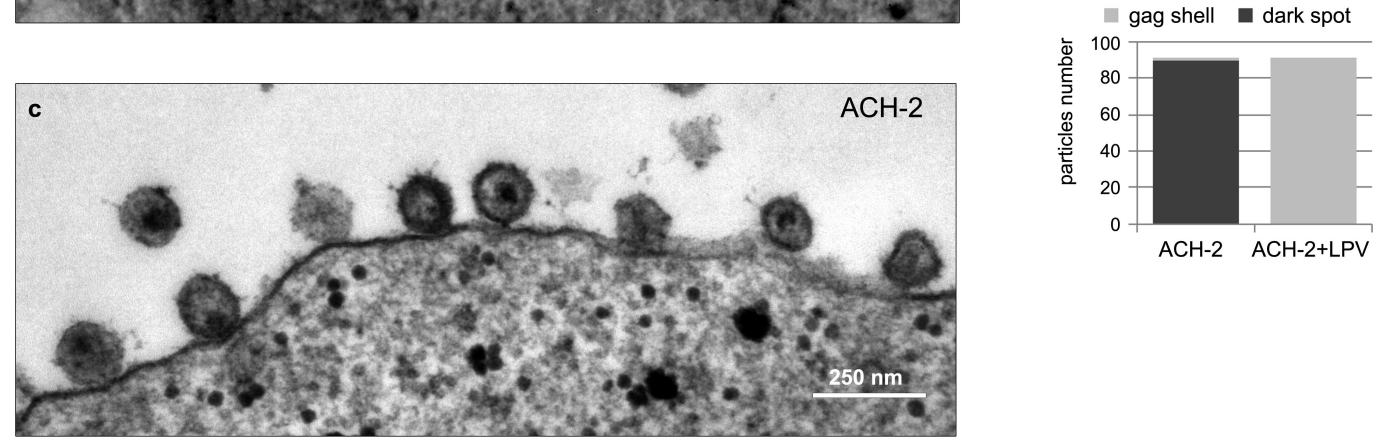

Figure 5 | Nucleocapsid condensation within HIV-1 particles depends on NCp15 processing and is detectable in membrane-attached particles. a, By TEM, HIV-1 $1_{\mathrm{NL} 4-3}$ virions accumulating NCp15 present defects in nucleocapsid condensation while NCp9- and NCp7containing viruses show correct core condensation into electron-dense dark spot. Quantitation was done for close to 180 counted particles. b-c, The majority of membrane-attached HIV-1 particles produced by latently infected $\mathrm{ACH}-2$ cells are immature particles in presence of LPV (b), they contain an electron-dense dark spot indicative of nucleocapsid condensation without LPV (c). Quantitation was done for close to 200 particles for LPV-treated ACH2 cells and to 1000 particles for non-treated ACH2 cells (d). 


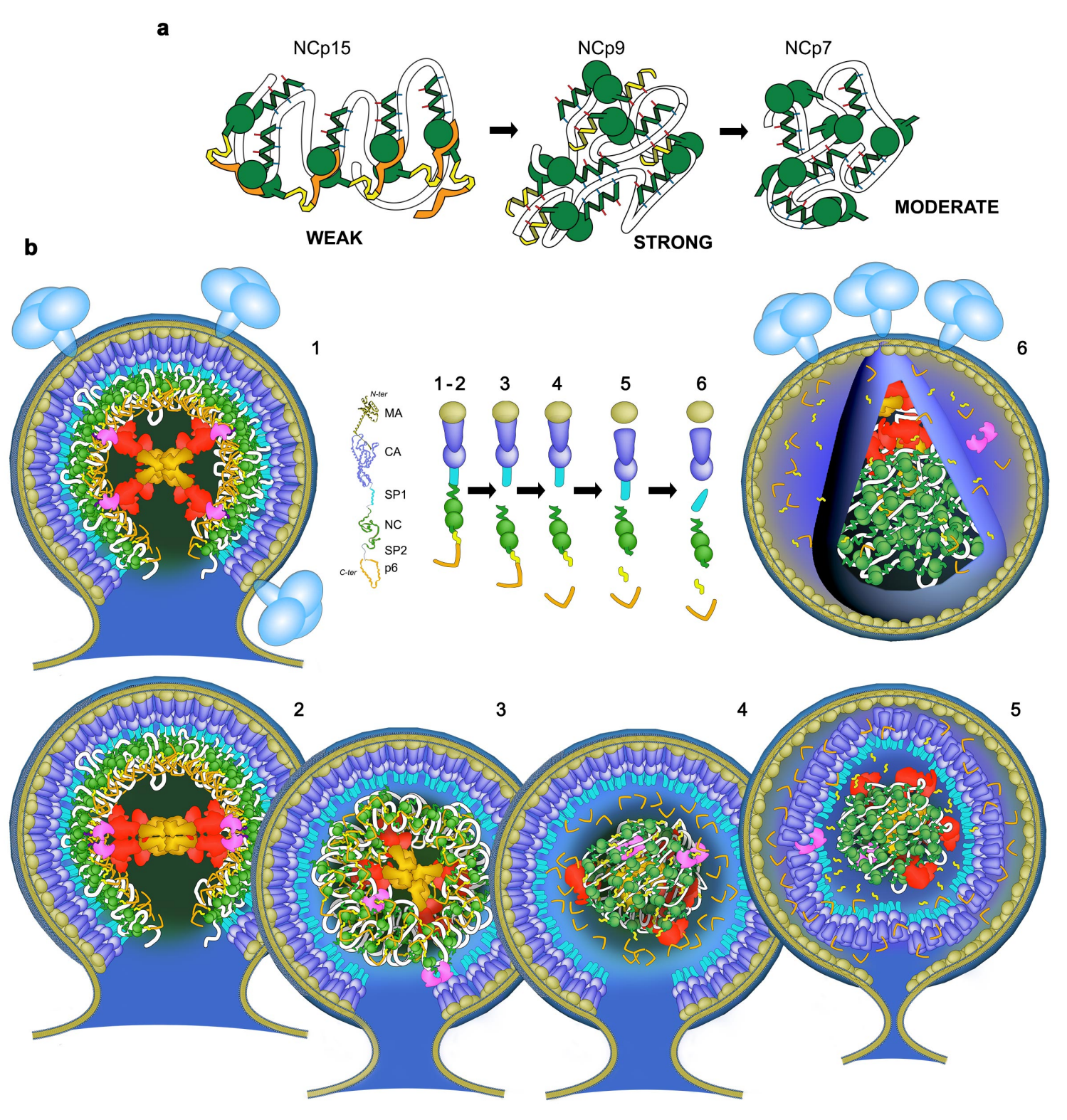

Figure 6 | Nucleocapsid condensation, quinary interactions and WSM transition of the nucleocapsid during HIV maturation. a, Weak-Strong-Moderate gluing properties of NC proteins with RNA (white) throughout virus maturation. p6 (orange)-NCp7 (green) contacts assemble NCp15 networks upon the RNA, blocking exposed residues engaged in NCp9 or NCp7 : NA contacts. Cleavage of p6 unmasks the NCp9 domain, the NCp7-SP2 (yellow) domain engages NC:NA:NC and NA:NC:NA networks favouring strong RNA-gluing and RNP granulation. Cleavage of SP2 removes one "K/F/Q" NA binding patch (see Fig 2a), which reduces the quinary network between RNA and NCp7. b, Nucleocapsid WSM transition in the context of viral particle. (1) Virus particle at the plasma membrane bud from self-assembly of Gag and 
GagPol on gRNA, with the NC domain as the RNA binder. The gRNA is shown in white within the layer of NC domains, assembled as bundles of six at the base of Gag hexamers. (2) The dimerization of GagPol self-activates PR (pink) and initiates maturation. (3) The SP1-NC site is cleaved, liberating the gRNA:NCp15 RNP, which in turns sequesters PR. (4) Rapid cleavage of NCp15 into NCp9, which liberates p6, unlocks the strong gluing properties of NCp9. This quickly compacts the gRNA and favours viral budding. gRNA condensation allows internal reorganization of RT (red) and IN (gold), self-assembly of the conical capsid after separation of MA from CA-SP1 and maturation of CA-SP1 while NCp9 are matured to NCp7 in (5 \& $\mathbf{6})$. 